\title{
Shannon entropy for stationary processes and dynamical systems
}

\author{
By D. Hamdan, W. Parry† and J.-P. Thouvenot
}

July 6,2021

\begin{abstract}
We consider stationary ergodic processes indexed by $\mathbb{Z}$ or $\mathbb{Z}^{n}$ whose finite dimensional marginals have laws which are absolutely continuous with respect to Lebesgue measure. We define an entropy theory for these continuous processes, prove an analog of the Shannon Breiman Mac Millan theorem and study more precisely the particular example of Gaussian processes.
\end{abstract}

\section{Introduction}

In [17] Shannon introduced a general theory of entropy designed to quantify the rate at which information is produced through the evolution of a stationary stochastic process. He gave two definitions: one for random variables assuming discrete values and the other for random variables which assume real values or, more generally, values in $\mathbb{R}^{n}$. However these definitions represent but two aspects of a single notion.

In 1958 Kolmogorov adapted Shannon's discrete version in his definition of entropy for dynamical systems which enabled him to solve an important open problem concerning the classification of measure-preserving transformations with continuous (in fact Lebesgue) spectrum. Shortly afterwards Sinai modified and improved Kolmogorov's definition so that today one speaks of the Kolmogorov-Sinai, K.S., invariant. 
The K.S. entropy is also an invariant for stationary stochastic processes in as much as they may be represented as measure preserving transformations. However these processes frequently have infinite entropy particularly when the random variables take their values in a non-discrete space. For example all stationary Gaussian processes with absolutely continuous spectrum have the same infinite K.S. entropy and indeed they are all measure theoretically isomorphic to each other (Ornstein [9]).

For this reason we feel that a modification of the traditional definition of isomorphism should be considered which distinguishes between various processes with infinite K.S. entropy. This paper should be regarded as a move toward this end in that we produce an «invariant ${ }^{»}$ based on Shannon's second version of entropy (designed for continuous valued random variables). Shannon's entropy was extensively investigated, especially by the Russian school, in the late 1950's and a thorough account appears in Pinsker's book [14]. One of our main purposes is to clarify and extend known results in this area.

The invariant investigated here ( naturally referred to as Shannon entropy ) is simply a normalised limit of entropy for $\mathbb{R}^{n}$ valued random variables, which we compute for all stationary Gaussian processes in terms of their spectral measures. In general this entropy is finite even when the K.S. entropy is infinite. We show how this entropy changes when a stationary Gaussian process is subjected to a linear transformation.

This is a topic closely related to the work of Wiener [20] and Kolmogorov [7] on linear prediction theory. It is well known that stationary Gaussian processes may be regarded as non-linear extensions of stationary linear sequences in Hilbert space (i.e. ${ }^{\ll}$ wide sense ${ }^{\gg}$ processes in the language of Doob [2] ) and entropy theory may be regarded as the prediction theory of non-linear processes. Wiener was particularly interested in non-linear prediction and in [21] he attempted to prove that all stationary processes satisfying certain mild conditions are isomorphic to independent Gaussian processes. As shown by Rosenblatt [15] his proof was flawed and it is interesting to note that his mistake, which concerned the behaviour of decreasing sequences of sigma algebras, was repeated by Kolmogorov (as shown By Rokhlin) in a different context some years later. We now know through the work of Ornstein and his co-workers that Wiener's claim is actually false.

We shall be concerned almost exclusively with stationary stochastic processes $X=\left(X_{n}\right)$ for which the distribution measure of $\left(X_{0}, \ldots, X_{n}\right)$ is absolutely continuous with respect to $n+1$ dimen- 
sional Lebesgue measure for all $n=0,1, \ldots$ Our first theorem shows that for any ergodic measurepreserving transformation $T$ of a probability space there exist functions $F$ such that $\left(F \circ T^{n}\right)$ satifies the above condition. We prove thereafter that an entropy « à la Kolmogorov» can be defined using the continuous entropy definition of Shannon. We prove that these averages of entropies always converge. The theory extends naturally to $\mathbb{Z}^{n}$ actions. In this framework, we prove a Shannon Mac Millan Breiman type pointwise theorem. However, if the Kolmogorov-Sinai entropy of the transformation is finite, then the limit above is always $-\infty$. In general, this limit is majorized by $\frac{1}{2} \log (2 \pi)$ plus one half of the variance of the observable. In particular, the preceding inequality reduces to equality if and only if the process is Gaussian independent ( This generalizes to processes a result of Shannon for the case of random variables). We give also a similar characterization for a stationary process to be Markovian, and, more generally, to be with memory $p$. The theory applies naturally to Gaussian processes, for which, we give a closed formula for this continuous entropy. It turns out that, in the Gaussian Markovian case, this entropy determines the process, and in the Gaussian case, when finite it determines the process up to unilateral isomorphism.

We give also some relationships with the rate of entropy of Pinsker and with the rate of generation of information as well.

William Parry, our co-author, died August 20-th 2006, at the time where these notes were completed.

\section{Absolutely continuous processes based on an ergodic system}

We begin with

Definition 2.1:

A real valued discrete time process $X=\left(X_{n}\right)_{n \in I}$, indexed by a countable set $I$, is said to be absolutely continuous if for every finite subset $K$ of $I$, with cardinality $|K|$, the joint distribution of $\left(X_{n}\right)_{n \in K}$ is absolutely continuous with respect to the $|K|$-dimensional Lebesgue measure.

We shall only be using $I=\mathbb{Z}$, or $I=\mathbb{Z}^{2}$.

In this section we prove the following:

Proposition 2.2: 
Let $(\Omega, T, \mu)$ be an invertible ergodic dynamical system. Then there exists $F \in L^{2}(\mu)$ such that the process $\left(F \circ T^{n}\right)$ is absolutely continuous.

Proof: Let $(Y, S, \gamma)$ be the independent gaussian dynamical system: $Y:=\mathbb{R}^{\mathbb{Z}}, S$ the shift transformation: $(S y)_{j}=y_{j+1}$, for $y \in Y, j \in \mathbb{Z}$, and $\gamma$ the product measure of the measures $\gamma_{j}, j \in \mathbb{Z}$, where, for every $j, \gamma_{j}=(2 \pi)^{-\frac{1}{2}} \exp \left(-\frac{1}{2} x^{2}\right) d l(x), l$ being the Lebesgue measure on $\mathbb{R}$. Let $Y_{n}: Y \rightarrow \mathbb{R}$ be the projection onto the $n$ 'th coordinate.

According to Dye's Theorem, [3], there exists an integer-valued measurable function $\tau: Y \rightarrow \mathbb{Z}$ such that if $S_{1}: Y \rightarrow Y$ is the transformation defined by $S_{1}(y)=S^{\tau(y)}(y)$, for $\gamma$-almost all $y \in Y$, then the two dynamical systems $(\Omega, T, \mu)$ and $\left(Y, S_{1}, \gamma\right)$ are isomorphic. Let $\theta: \Omega \rightarrow Y$ be a map giving the isomorphism: $\theta \circ T=S_{1} \circ \theta$ and $\gamma=\mu \circ \theta^{-1}$. Set $F=Y_{0} \circ \theta$ and $Z_{n}=Y_{0} \circ S_{1}^{n}$, for $n \in \mathbb{Z}$, so that $\left(F, F \circ T, \ldots, F \circ T^{n-1}\right)$ and $\left(Z_{0}, Z_{1}, \ldots Z_{n-1}\right)$ have the same law, say $\alpha_{n}$. We show now that $\alpha_{n}$ is absolutely continuous with respect to Lebesgue measure and this ends the proof. To do this, for every $\bar{k}=\left(k_{1}, \ldots, k_{n-1}\right) \in \mathbb{Z}^{n-1}$, put

$$
E_{\bar{k}}=\left\{\tau=k_{1}, \tau \circ S^{k_{1}}=k_{2}, \tau \circ S^{k_{1}+k_{2}}=k_{3}, \ldots, \tau \circ S^{k_{1}+\ldots+k_{n-2}}=k_{n-1}\right\},
$$

and let $\mathcal{F}_{\bar{k}}$ denote the sigma-algebra generated by $Y_{0}, Y_{k_{1}}, \ldots, Y_{k_{1}+\ldots+k_{n-1}}$. In view of the definition of $(Y, S, \gamma)$, straightforeward computations show then that $\alpha_{n}$ is absolutely continuous with respect to Lebesgue measure and has the density $g:=\sum_{\bar{k} \in Z^{n}} g_{\bar{k}}$, where

$$
g_{\bar{k}}\left(y_{0}, \ldots, y_{n-1}\right)=E_{\gamma}\left[1_{E_{\bar{k}}} \mid \mathcal{F}_{\bar{k}}\right]\left(y_{0}, y_{1}, \ldots, y_{n-1}\right) \times(2 \pi)^{-\frac{n}{2}} \exp \left(-\frac{1}{2}\left(y_{0}^{2}+\ldots+y_{n-1}^{2}\right)\right)
$$

and $E_{\gamma}\left[1_{E_{\bar{k}}} \mid \mathcal{F}_{\bar{k}}\right]$ denotes the conditional expectation with respect to $\gamma$ of $1_{E_{\bar{k}}}$ given the sigma algebra $\mathcal{F}_{\bar{k}}$.

\section{Remark 2.3:}

(1) The function $F$ in Lemma 2 can be taken ( as the proof shows ) in the intersection of $\left\{L^{p}(\mu)\right.$ : $p \geq 1\}$.

(2) We can show that there is $F$ such that, for every $n$, the law of $\left(F, F \circ T, \ldots, F \circ T^{n-1}\right)$ is equivalent to the $n$-dimensional Lebesgue measure.

In the same way we have 


\section{Proposition 2.4:}

If $T$ and $S$ are measure preserving transformations which commute on a probability space $(\Omega, \mathcal{F}, \mu)$, such that the joint action is ergodic then there exists $F \in L^{2}(\mu)$ such that the process $\left(F \circ T^{m} \circ\right.$ $\left.S^{n}\right)_{(m, n) \in \mathbb{Z}^{2}}$ is absolutely continuous.

\section{Notations, a few prerequisites and a lemma}

\subsection{Conditional entropy of probability measures}

In this subsection we recall various definitions attached to Shannon entropy [14 ].

\section{Definition 3.1 :}

Let $P$ and $Q$ be probability measures defined on the same measurable space $(\Omega, \mathcal{F})$. Let $\mathcal{P}$ be the set of all finite measurable partitions of $\Omega$. If $\Pi$ is in $\mathcal{P}$ let

$$
S_{\Pi}(P \mid Q):=\sum_{E \in \Pi} P(E) \log \left(\frac{P(E)}{Q(E)}\right) .
$$

Then for $\Pi_{1} \in \mathcal{P}, \Pi_{1}$ finer than $\Pi$ implies

$$
S_{\Pi}(P \mid Q) \leq S_{\Pi_{1}}(P \mid Q)
$$

The entropy $H_{Q}(P)$ of $P$ with respect to $Q$ is defined by

$$
H_{Q}(P):=\sup _{\Pi \in \mathcal{P}} S_{\Pi}(P \mid Q)
$$

We list without proofs some results which we are going to use.

Theorem A (Gelfand, Yaglom, Perez, [14]): Let $P, Q$ be two probabiliy measures on the 
measurable space $(\Omega, \mathcal{F})$. Then

If the entropy $H_{Q}(P)$ is finite then $P$ is absolutely continuous with respect to $Q$ and

$$
H_{Q}(P)=\int \log \left(\frac{d P}{d Q}\right) d P
$$

(In particular if $P$ is not absolutely continuous with respect to $Q, H_{Q}(P)=+\infty$ ).

This was introduced first by Shannon [ 17 ], for densities:

If $f \in L_{+}^{1}(d x)$ is such that $\int_{\mathbb{R}} f(x) d x=1$, Shannon considered $\int_{\mathbb{R}} f(x) \log f(x) d x$.

Let $\psi$ be the function defined for $x>0$, by

$$
\psi(x)=-x \log (x)
$$

\section{Remark 3.2:}

(1) If $H_{Q}(P)$ is finite then

$$
H_{Q}(P)=\int \log \left(\frac{d P}{d Q}\right) \times \frac{d P}{d Q} d Q=-\int \psi\left(\frac{d P}{d Q}\right) d Q
$$

In particular,

(2) $H_{Q}(P)=0$ if and only if $P=Q$.

(3) More generally, If $P$ is absolutely continuous with respect to $Q$ then

$$
H_{Q}(P)<\infty \Longleftrightarrow \int \log \left(\frac{d P}{d Q}\right) d P<\infty \Longleftrightarrow \int(-\psi)\left(\frac{d P}{d Q}\right) d Q<\infty
$$

The following theorem follows from the monotonicity property (2).

Theorem B (Dobrushin, [14]): Let $P, Q$ be probability measures on $(\Omega, \mathcal{F}), \mathcal{L}$ an algebra of sets belonging to $\mathcal{F}$, which generates the sigma-algebra $\mathcal{F}$, and let $\mathcal{R}$ be a family of finite partitions of $\Omega$ whose elements belong to $\mathcal{L}$. If every partition consisting of sets from $\mathcal{L}$ has a finer partition in 
$\mathcal{R}$, then

$$
H_{Q}(P)=\sup _{\Pi \in \mathcal{R}} S_{\Pi}(P \mid Q)
$$

* Note that, as remarked by the translator of Pinsker, in Theorem B of Dobrushin, the condition that the elements of the partitions in $\mathcal{R}$ be in $\mathcal{L}$ is not necessary.

\section{$3.2 \quad$ A lemma}

The following lemma will play an essential role in the rest of the paper.

\section{Lemma 3.3:}

Let $\left(\Omega_{i} ; \mathcal{F}_{i}, P_{i}\right)$ be a probability space for $i=1,2$, and $\nu$ a probability measure on $\left(\Omega_{1} \times \Omega_{2}, \mathcal{F}_{1} \otimes \mathcal{F}_{2}\right)$ with marginals $\nu_{1}$ on $\left(\Omega_{1}, \mathcal{F}_{1}\right)$ and $\nu_{2}$ on $\left(\Omega_{2}, \mathcal{F}_{2}\right)$. Then

$$
H_{P_{1} \times P_{2}}(\nu)=H_{\nu_{1} \times \nu_{2}}(\nu)+H_{P_{1}}\left(\nu_{1}\right)+H_{P_{2}}\left(\nu_{2}\right),
$$

and in particular

$$
H_{P_{1} \times P_{2}}(\nu) \geq H_{P_{1}}\left(\nu_{1}\right)+H_{P_{2}}\left(\nu_{2}\right),
$$

$$
H_{P_{1} \times P_{2}}(\nu) \geq H_{\nu_{1} \times \nu_{2}}(\nu) .
$$

Proof: By Theorem B of Dobrushin, $H_{P_{1} \times P_{2}}(\nu)$ is given by the supremum, over all finite measurable partitions $\Pi_{1}$ of $\Omega_{1}$ and $\Pi_{2}$ of $\Omega_{2}$, of the sums $S_{\Pi_{1} \times \Pi_{2}}\left(\nu \mid P_{1} \times P_{2}\right)$.

If $\nu$ is not absolutely continuous with respect to $\nu_{1} \times \nu_{2}$, then, by Theorem $\mathrm{A}, H_{\nu_{1} \times \nu_{2}}(\nu)=+\infty$, and thus

$$
H_{P_{1} \times P_{2}}(\nu) \leq+\infty=H_{\nu_{1} \times \nu_{2}}(\nu)+H_{P_{1}}\left(\nu_{1}\right)+H_{P_{2}}\left(\nu_{2}\right)
$$

If $\nu$ is absolutely continuous with respect to $\nu_{1} \times \nu_{2}$, the equalities

$$
\nu(E \times F) \log \frac{\nu(E \times F)}{P_{1} \times P_{2}(E \times F)}=\nu(E \times F)\left[\log \frac{\nu(E \times F)}{\nu_{1} \times \nu_{2}(E \times F)}+\log \frac{\nu_{1}(E)}{P_{1}(E)}+\log \frac{\nu_{2}(F)}{P_{2}(F)}\right], E \in \Pi_{1}, F \in \Pi_{2}
$$


imply

$$
S_{\Pi_{1} \times \Pi_{2}}\left(\nu \mid P_{1} \times P_{2}\right)=S_{\Pi_{1} \times \Pi_{2}}\left(\nu \mid \nu_{1} \times \nu_{2}\right)+S_{\Pi_{1}}\left(\nu_{1} \mid P_{1}\right)+S_{\Pi_{2}}\left(\nu_{2} \mid P_{2}\right), \quad\left(E_{1}\right)
$$

and therefore

$$
S_{\Pi_{1} \times \Pi_{2}}\left(\nu \mid P_{1} \times P_{2}\right) \leq H_{\nu_{1} \times \nu_{2}}(\nu)+H_{P_{1}}\left(\nu_{1}\right)+H_{P_{2}}\left(\nu_{2}\right),
$$

from which it follows that

$$
H_{P_{1} \times P_{2}}(\nu) \leq H_{\nu_{1} \times \nu_{2}}(\nu)+H_{P_{1}}\left(\nu_{1}\right)+H_{P_{2}}\left(\nu_{2}\right)
$$

To prove the reverse inequality

$$
H_{P_{1} \times P_{2}}(\nu) \geq H_{\nu_{1} \times \nu_{2}}(\nu)+H_{P_{1}}\left(\nu_{1}\right)+H_{P_{2}}\left(\nu_{2}\right), \quad\left(E_{2}\right)
$$

consider four arbitrary finite measurable partitions: $\Pi_{1}, \Delta_{1}$ of $\Omega_{1}$, and $\Pi_{2}, \Delta_{2}$ of $\Omega_{2}$, and note that we can find a finite measurable partition $\Gamma_{i}$ of $\Omega_{i}$ refining both $\Pi_{i}$ and $\Delta_{i}, i=1,2$. Then the partition $\Gamma_{1} \times \Gamma_{2}:=\left\{M \times N: M \in \Gamma_{1}, N \in \Gamma_{2}\right\}$ refines also $\Pi_{1} \times \Pi_{2}$. But, in view of inequality (2), we have, for $i=1,2$,

$$
S_{\Delta_{i}}\left(\nu_{i} \mid P_{i}\right) \leq S_{\Gamma_{i}}\left(\nu_{i} \mid P_{i}\right)
$$

Similarly

$$
S_{\Pi_{1} \times \Pi_{2}}\left(\nu \mid \nu_{1} \times \nu_{2}\right) \leq S_{\Gamma_{1} \times \Gamma_{2}}\left(\nu \mid \nu_{1} \times \nu_{2}\right) .
$$

So by summing we get, by $\left(E_{1}\right)$

$$
\left.S_{\Delta_{1}}\left(\nu_{1} \mid P_{1}\right)+S_{\Delta_{2}}\left(\nu_{2} \mid P_{2}\right)+S_{\Pi_{1} \times \Pi_{2}}\left(\nu \mid \nu_{1} \times \nu_{2}\right) \leq S_{\Gamma_{1} \times \Gamma_{2}}\left(\nu \mid P_{1} \times P_{2}\right)\right) \leq H_{P_{1} \times P_{2}}(\nu),
$$

from which $\left(E_{2}\right)$ follows. This proves (7). As trivially (7) implies (8) and (9), the proof is finished. 


\section{Remark 3.4:}

The inequality (9) implies that, for fixed $\nu$, the infimum, over all probability measures $P_{1}$ and $P_{2}$, of $H_{P_{1} \times P_{2}}(\nu)$ is attained for $P_{1}=\nu_{1}$ and $P_{2}=\nu_{2}$, and the formula (7) shows that it is attained only for these particular values of $P_{1}$ and $P_{2}$.

\section{Corollary 3.5:}

Let $\nu$ be a probability measure on a product measurable space. If the entropy $H_{P_{1} \times P_{2}}(\nu)$ of $\nu$, with respect to a product probability measure $P_{1} \times P_{2}$, is finite, then $\nu$ is absolutely continuous with respect to the product $\nu_{1} \times \nu_{2}$ of its marginals, and these marginals are absolutely continuous with respect to $P_{1}$ and $P_{2}$ respectively.

\section{Shannon entropy of absolutely continuous processes}

\subsection{Notation}

Let $(\Omega, T, \mu)$ be an invertible ergodic dynamical system. Let $\mathcal{A}=\left\{A_{1}, \ldots, A_{k}\right\}$ be a finite partition of $\Omega$. Let $m$ on $\mathcal{A}^{\mathbb{Z}}$ be the product measure with marginals giving equal weights to the atoms of $\mathcal{A}$. Let $F=\sum_{j=0}^{k} a_{j} 1_{A_{j}}$ be discrete with $a_{i} \neq a_{j}$ for $i \neq j$, and $F_{n}(x)=\left(F(x), \ldots, F\left(T^{n-1} x\right)\right)$, for

$x \in \Omega$. Then if $\mu_{n}=\mu F_{n}^{-1}$ and $m_{n}$ respectively are the restrictions of $\mu$ and $m$ to $\bigvee_{j=0}^{n-1} T^{j} \mathcal{A}$, we obtain, with the standard definition of entropy of a partition

$$
H^{\mu}\left(\bigvee_{j=0}^{n-1} T^{-j} \mathcal{A}\right)=-\int_{\Omega} \log \frac{d \mu F_{n}^{-1}}{d m_{n}} \circ F_{n} d \mu
$$

In this paper we are interested in the case where $F$ is continuous valued, say real valued, with $\left(F \circ T^{n}\right)$ absolutely continuous (cf. Definition 2.1). In this case, denoting $l^{n}$ the Lebesgue measure 
on $\mathbb{R}^{n}$, we consider the function $\mathcal{I}_{n}=\mathcal{I}_{n}(F)$ defined by:

$$
\mathcal{I}_{n}(x):=-\log \left(\frac{d \mu F_{n}^{-1}}{d l^{n}}\right) \circ F_{n}(x), \quad x \in \Omega
$$

together with its integral

$$
H_{n}=H_{n}(F):=\int_{\Omega} \mathcal{I}_{n} d \mu
$$

Then, with $\psi$ as in (4), it follows

$$
H_{n}(F)=\int_{\mathbb{R}^{n}} \psi\left(\frac{d \mu F_{n}^{-1}}{d l^{n}}\right) d l^{n} .
$$

We focus on the asymptotic behavior of the sequences $\frac{1}{n} \mathcal{I}_{n}$ and $\frac{1}{n} H_{n}$, which we call, respectively, the sequences of Shannon information and Shannon entropy associated to the process $\left(F \circ T^{n}\right)$. Let

$$
\gamma_{0}=\frac{1}{\sqrt{2 \pi}} \exp \left(-\frac{1}{2} x^{2}\right) d l(x), \quad \gamma_{n}=\gamma_{0}^{\otimes n}, n \geq 1
$$

The following quantities are closely related to $\mathcal{I}_{n}$ and $H_{n}$ :

$$
\begin{gathered}
\mathcal{I}_{n, G}=\mathcal{I}_{n, G}(F)=-\log \frac{d \mu F_{n}^{-1}}{d \gamma_{n}} \circ F_{n} \quad\left(H_{n, G}=H_{n, G}(F)=\int \mathcal{I}_{n, G}(F) d \mu\right), \\
\mathcal{I}_{n, P M}=\mathcal{I}_{n, P M}(F)=-\log \frac{d \mu F_{n}^{-1}}{d\left(\mu F^{-1}\right)^{\otimes n}} \circ F_{n} \quad\left(H_{n, P M}(F)=\int \mathcal{I}_{n, P M}(F) d \mu\right) .
\end{gathered}
$$

The link between these quantities, which behave very much the same, is made precise in the formulas (46) and (47).

We shall employ each of the above quantities as seems appropriate. It should be clear that a result formulated using one is easily transformed into a result formulated in terms of the other.

In the case of ergodic $\mathbb{Z}^{2}$ action we shall use the following notation

If $F: \Omega \rightarrow \mathbb{R}$ is measurable and $K$ is a finite subset of $\mathbb{Z}^{2}$, let $F_{K}(x)=\left(F\left(T^{m} S^{n} x\right)\right)_{(m, n) \in K}$, 
for $x \in \Omega$. If the law of $F_{K}$ is absolutely continuous with respect to $|K|$-dimensional Lebesgue measure, we denote $f_{K}$ its density. In particular if $K=\left\{(i, j) \in \mathbb{Z}^{2}: 0 \leq i \leq n-1,0 \leq j \leq n-1\right\}$, we denote $F_{K}$ by $F_{n^{2}}$ and $f_{K}$ by $f_{n^{2}}$. If the process $\left(F \circ T^{m} \circ S^{n}\right)_{(m, n) \in \mathbb{Z}^{2}}$ is absolutely continuous, as in one dimensional case, we consider

$$
h_{n}^{(2)}:=-\log f_{n^{2}} \circ F_{n^{2}},
$$

and its integral

$$
H_{n}^{(2)}:=\int h_{n}^{(2)} d \mu
$$

As our concern is the asymptotic behavior of $\frac{1}{n^{2}} h_{n}^{(2)}$, there is no loss of generality if we suppose that $\Omega=\mathbb{R}^{\mathbb{Z}^{2}}, F$ is the projection onto the zero coordinate, $(T x)_{g}=x_{g+(0,1)},(S x)_{g}=x_{g+(1,0)}$, for $x \in \mathbb{R}^{\mathbb{Z}^{2}}, g \in \mathbb{Z}^{2}$, and that $\mu$ is a probability measure whose finite dimensional marginals are absolutely continuous with respect to Lebesgue measure, and which is invariant by the shifts $T$ and $S$. In this case all the densities $f_{n^{2}}$, for various $n$, will be denoted by $f$ without subscript, so that for every $x \in \mathbb{R}^{\mathbb{Z}^{2}}$, we have

$$
\frac{1}{n^{2}} h_{n}^{(2)}(x)=-\frac{1}{n^{2}} \log f\left(X_{n-1, n-1}^{n}\right),
$$

where

$$
X_{i, j}^{n}:=\left(x_{s, t}\right)_{(s, t) \in I_{i, j}^{n}, \quad\left(\alpha_{0}\right)}
$$

and

$$
I_{i, j}^{n}:=\{(s, j): s=0, \ldots, i\} \cup\{(s, t): 0 \leq s \leq n-1,0 \leq t \leq j-1\}
$$

for $1 \leq i, j \leq n-1, I_{0,0}^{n}=\{(0,0)\}$ and $I_{i, 0}^{n}=\{(s, 0): s=0, \ldots, i\}$. Let, for future use,

$$
Y_{i, j}^{n}:=\left(x_{s, t}\right)_{(s, t) \in I_{i, j}^{n},(s, t) \neq(0,0)} .
$$


In particular

$$
X_{n, n}^{n+1}:=\left(x_{s, t}\right)_{s, t=0, \ldots, n}
$$

and

$$
Y_{n, n}^{n+1}:=\left(x_{s, t}\right)_{s, t=0, \ldots, n,(s, t) \neq 0}
$$

Let

$$
L:=\{(i, j, n): 0 \leq i, j \leq n-1, n \geq 1\}
$$

The set inclusion on the $I_{i, j}^{n}$, induces a partial order on $L$ : we set

$$
(i, j, n) \leq\left(i^{\prime}, j^{\prime}, n^{\prime}\right) \Longleftrightarrow I_{i, j}^{n} \subset I_{i^{\prime}, j^{\prime}}^{n^{\prime}} \Longleftrightarrow\left(j<j^{\prime}, n \leq n^{\prime}\right) \operatorname{or}\left(j=j^{\prime}, i \leq i^{\prime}, n \leq n^{\prime}\right) .
$$

That is the product of the lexicographical order on $\{(j, i)\}$ and the usual order on $\{n\}$.

In the remark below, we single out two properties which we use later:

\section{Remark 4.1:}

(1) $L$ is directed, and $C:=\{n-1, n-1, n): n \geq 1\}$ is a cofinal subset of $L$.

(2) if $\left\{\left(i_{k}, j_{k}, n_{k}\right): k \in \mathbb{N}\right\}$ is an infinite subset of $L$, then there is an infinite subset $J$ of $\mathbb{N}$, such that the sequence $\left(\left(i_{k}, j_{k}, n_{k}\right)\right)_{k \in J}$ is strictly increasing in $L$, and $\lim _{k \in J} n_{k}+\infty$.

For $l=(i, j, n) \in L$, denote $\mathcal{F}_{l}$ or $\sigma\left(X_{i, j}^{n}\right)$, the sigma-algebra generated by $\left\{x_{s, t}:(s, t) \in I_{i, j}^{n}\right\}$. Then, for $l, l^{\prime} \in L, l \leq l^{\prime} \Longleftrightarrow \mathcal{F}_{l} \subset \mathcal{F}_{l^{\prime}}$.

If $m$ is a probability measure on $\mathbb{R}^{\mathbb{N}^{2}}, m_{i, j}^{n}$ will denote its restriction to $\mathcal{F}_{l}=\sigma\left(X_{i, j}^{n}\right)$, and then we write

$$
m_{i, j}^{n}:=m \mid \sigma\left(X_{i, j}^{n}\right)
$$

and in particular $m_{n-1, n-1}^{n}$ will be denoted simply $m_{n}$.

Two particular probability measures $\pi$ and $\nu$ on $\mathbb{R}^{\mathbb{N}^{2}}$, will be useful for our purpose. Their finite dimensional marginals $\pi_{n}=\pi_{n-1, n-1}^{n}$ and $\nu_{n}=\nu_{n-1, n-1}^{n}$ are given by

$$
\pi_{n}=\prod_{(s, t) \in I_{n-1, n-1}^{n}} f\left(x_{s, t}\right) d \lambda
$$


and

$$
\nu_{n}=f\left(x_{0,0}\right) \times f\left(Y_{n-1, n-1}^{n}\right) d \lambda
$$

where $Y_{n-1, n-1}^{n}$ is as in $\left(a_{1}\right)$ and $\lambda=\lambda_{n^{2}}$ denotes $n^{2}$-dimensional Lebesgue measure.

Recall also that

$$
\mu_{n}=f\left(X_{n-1, n-1}^{n}\right) d \lambda
$$

where $X_{n-1, n-1}^{n}$ is as in $\left(a_{0}\right)$.

\subsection{Convergence of the Shannon entropy}

We now turn to the dynamical situation. We consider a dynamical system $(\Omega, T, \mu)$ and denote $A C(\Omega, T, \mu)$ the set of functions $F \in L^{2}(\mu)$ such that the process $\left(F \circ T^{n}\right)$ is absolutely continuous (i.e. as in Definition 2.1).

We establish the convergence of the sequence of Shannon entropy $\frac{H_{n}(F)}{n}$ defined by (11), and give, as in the discrete valued case, an a priori upper bound for this limit and a criterion implying that the process $\left(F \circ T^{n}\right)$ is Gaussian independent (Corollary 4.5). We also identify this limit ( Lemma $4.8)$.

In the same way, for $\mathbb{Z}^{2}$ action, with $T$ and $S$ as generators, $A C(\Omega, T, S, \mu)$ will denote the set of $F \in L^{2}(\mu)$ such that the process $\left(F \circ T^{m} S^{n}\right)_{m, n \in \mathbb{Z}}$ is absolutely continuous (i.e. Definition 2.1).

The next formula gives one of the announced links.

Recall that $\gamma_{n}$ denotes the independent Gaussian measure ( cf. (13) ), and $\psi$ is as in (4).

\section{Lemma 4.2:}

Let $F \in A C(\Omega, T, \mu)$. Then

$$
\frac{H_{n}(F)}{n}=\frac{1}{n} \int_{\mathbb{R}^{n}} \psi\left(\frac{d \mu F_{n}^{-1}}{d \gamma_{n}}\right) d \gamma_{n}+\frac{1}{2} \log (2 \pi)+\frac{1}{2}\|F\|_{2}^{2} .
$$


Proof: From formula (12), we get

$$
H_{n}=\int \frac{d \mu F_{n}^{-1}}{d \gamma_{n}} \psi\left(\frac{d \gamma_{n}}{d l^{n}}\right) d l^{n}+\int \psi\left(\frac{d \mu F_{n}^{-1}}{d \gamma_{n}}\right) d \gamma_{n}
$$

But, if $h(t):=\frac{1}{\sqrt{2 \pi}} \exp \left(-\frac{1}{2} t^{2}\right)$ for $t \in \mathbb{R}$, then

$$
\begin{array}{r}
\int \frac{d \mu F_{n}^{-1}}{d \gamma_{n}} \psi\left(\frac{d \gamma_{n}}{d l^{n}}\right) d l^{n}=\int-\log \frac{d \gamma_{n}}{d l^{n}} d \mu F_{n}^{-1}=-\sum_{j=0}^{n-1} \int \log \left(h\left(x_{j}\right)\right) d \mu F_{n}^{-1}(x)=-\sum_{j=0}^{n-1} \int \log \left(h\left(F\left(T^{j} x\right)\right)\right) d \mu(x) \\
=-n \int \log (h(F(x))) d \mu(x)=-n\left[-\frac{1}{2} \log (2 \pi)-\frac{1}{2} \int F(x)^{2} d \mu(x)\right]=n \times\left[\frac{1}{2} \log (2 \pi)+\frac{1}{2}\|F\| \|_{2}^{2}\right] . \square
\end{array}
$$

\section{Remark 4.3:}

(i) The preceding formula (22) can be written as

$$
H_{n}(F)=-H_{\gamma_{n}}\left(\mu F_{n}^{-1}\right)+\frac{n}{2}\left(\log 2 \pi+\|F\|_{2}^{2}\right)
$$

(ii) If $H_{n}(F)$ is infinite then $H_{n+1}(F)$ is infinite.

In fact, $(i i)$ follows from $(i)$, and (i) from formula (5).

\section{Lemma 4.4:}

Let $F \in A C(\Omega, T, \mu)$. Then $\left(H_{n}(F)\right)_{n \in N}$ is a sub-additive sequence: for $n, p \in N$

$$
H_{n+p} \leq H_{n}+H_{p}
$$

Proof: Inequality (24) follows immediately from Lemma 3.3 and formula (23).

The following corollary is the analogue for the Shannon entropy of the fact that the Kolmogorv entropy of a countable states process is bounded by the entropy of the zerot'h coordinate. The 
equality case is analogous to the fact that in the Kolmogorov situation, the equality implies that the process is Bernoulli.

\section{Corollary 4.5:}

Let $(\Omega, T, \mu)$ be a dynamical system. Then for any $F \in A C(\Omega, T, \mu)$ the sequence $\left(\frac{H_{n}(F)}{n}\right)$ of Shannon entropies converges to $S e(F, T)$, which may be infinite. Moreover:

$$
\operatorname{Se}(F, T) \leq \frac{1}{2}\left(\log (2 \pi)+\|F\|_{2}^{2}\right)
$$

and the equality holds if and only if for every $n$ the law $\mu F_{n}^{-1}$ of $\left(F, F \circ T, \ldots, F \circ T^{n-1}\right)$ is the gaussian independent measure $\gamma_{n}$.

Proof: The sequence $\left(\frac{H_{n}}{n}\right)$ converges to its infimum, since $\left(H_{n}\right)$ is sub-additive by Lemma 4.4. On the other hand, formula (23) implies $\frac{H_{n}}{n} \leq \frac{1}{2}\left(\log (2 \pi)+\|F\|_{2}^{2}\right)$, which yields then the inequality $(25)$.

To prove the other statement, note that from formula $(23)$ and the equality $\operatorname{Se}(F, T)=\inf \left\{\frac{H_{n}}{n}\right.$ : $n \in N\}$, it follows that the equality in (25) is equivalent to the equalities $H_{\gamma_{n}}\left(\mu F_{n}^{-1}\right)=0, \forall n$. But this is equivalent to the equalities $\mu F_{n}^{-1}=\gamma_{n}, \forall n$ by Remark 3.2(2). $\square$

Note that the preceding corollary generalizes to processes a theorem of Shannon that among random variables with fixed variance the maximum of the continuous entropy is achieved by a gaussian variable.

A similar proof can yield an $n$ dimensional version of this theorem of Shannon .

\section{Proposition 4.6}

$$
\begin{gathered}
\text { Let } \mathcal{M}_{1}(n)=\left\{p \in L_{+}^{1}\left(l^{n}\right): \int_{\mathbb{R}^{n}} p(x) d l^{n}(x)=1, \int_{\mathbb{R}^{n}}\|x\|_{2}^{2} p(x) d l^{n}(x)=n\right\} . \text { Then } \\
\sup _{p \in \mathcal{M}_{1}(n)} \int_{\mathbb{R}^{n}} \psi(p(x)) d l^{n}(x)=\frac{n}{2}(1+\log (2 \pi)) .
\end{gathered}
$$


Furthermore this supremum is attained for the gaussian independent density

$$
p(x)=(2 \pi)^{-\frac{n}{2}} \exp \left(-\frac{1}{2}\left(x_{0}^{2}+\ldots+x_{n-1}^{2}\right)\right)
$$

for $x=\left(x_{0}, \ldots, x_{n-1}\right) \in \mathbb{R}^{n}$, and this is the only one.

We came to the main definition of this section:

\section{Definition 4.7:}

The Shannon entropy $S e(F, T)$ of the process $\left(F \circ T^{n}\right)$ is defined by the equality

$$
S e(F, T):=\lim _{n} \frac{H_{n}(F)}{n}
$$

Next we identify the limit in definition 4.7 , the Shannon entropy $S e(F, T)$ of the process $\left(F \circ T^{n}\right)$, using conditional entropy, or information ( Lemma 4.8 (b) (ii) and (iii)).

\section{Lemma 4.8:}

(a) Suppose that $H_{n+p}$ is finite. Then

$$
H_{\mu F_{n}^{-1} \times \mu F_{p}^{-1}}\left(\mu F_{n+p}^{-1}\right)=H_{n}+H_{p}-H_{n+p},
$$

and, for fixed $n$, the sequence $H_{\mu F_{n}^{-1} \times \mu F_{p}^{-1}}\left(\mu F_{n+p}^{-1}\right)$ is increasing in $p$.

(b) If $H_{n}$ is finite for all $n$, then

(i) $S e(F, T)$ is finite if and only if for every $n$, or for some $n, \sup _{p} H_{\mu F_{n}^{-1} \times \mu F_{p}^{-1}}\left(\mu F_{n+p}^{-1}\right)$ is finite.

$$
\sup _{p} H_{\mu F_{n}^{-1} \times \mu F_{p}^{-1}}\left(\mu F_{n+p}^{-1}\right)=H_{n}-n \times S e(F, T) .
$$

(iii) If $\nu$ is the law of the process $(F, F \circ T, \ldots)$ and $\nu_{n}$ is the probability measure on $\mathbb{R}^{\mathbb{N}}$, with 
$n+p$-marginal given by $\mu F_{n}^{-1} \times \mu F_{p}^{-1}$, for any $p \geq 0$, then

$$
H_{\nu_{n}}(\nu)=H_{n}-n S e(F, T)
$$

In particular, if $\operatorname{Se}(F, T)$ is finite, $\nu$ is abolutely continuous with respect to $\nu_{n}$.

Proof: (a) Formula (27) follows from Lemma 3.3 and formula (23). The other property follows from the definition, since, when $\Pi_{1}$ and $\Pi_{2}$ are finite partitions of $\mathbb{R}^{n}$ and $\mathbb{R}^{p}$, respectively, we have

$$
S_{\Pi_{1} \times \Pi_{2}}\left(\mu F_{n+p}^{-1} \mid \mu F_{n}^{-1} \times \mu F_{p}^{-1}\right) \leq S_{\Pi_{1} \times\left(\Pi_{2} \times \mathbb{R}\right)}\left(\mu F_{n+p+1}^{-1} \mid \mu F_{n}^{-1} \times \mu F_{p+1}^{-1}\right) .
$$

(b) Put $v_{p}^{n}:=H_{p}-H_{n+p}$, and in particular, for $n=1$,

$$
u_{p}:=-v_{p}^{1}=H_{p+1}-H_{p}
$$

Then, from (a) above, the sequence $\left(u_{p}\right)$ is decreasing. So, as $\frac{1}{N} \sum_{p=1}^{N} u_{p}$ converges to $\operatorname{Se}(F, T)$, $\left(u_{n}\right)$ converges also to $S e(F, T)=\inf _{p} u_{p}$. This proves (i). (ii) follows from (i) and the equality $v_{p}^{n}=-u_{p}-u_{p+1}-\ldots-u_{n+p-1}$. (iii) follows from $(i i)$.

\section{Remark 4.9:}

If $\operatorname{Se}(F, T)$ is finite then

$$
\lim _{n} \frac{1}{n} \sup _{p} H_{\mu F_{n}^{-1} \times \mu F_{p}^{-1}}\left(\mu F_{n+p}^{-1}\right)=0
$$

and for fixed $p, H_{\mu F_{n}^{-1} \times \mu F_{p}^{-1}}\left(\mu F_{n+p}^{-1}\right)$ is increasing in $n$, and $z_{n}:=\sup _{p} H_{\mu F_{n}^{-1} \times \mu F_{p}^{-1}}\left(\mu F_{n+p}^{-1}\right)$ is sub-additive and increasing.

For the convergence of Shannon entropy in the case of $\mathbb{Z}^{2}$ action, for $F \in A C(\Omega, T, S, \mu)$, we have the following

Lemma 4.10: 
Let $\mu_{n}$ and $\pi_{n}$ be as in (21) and (19) respectively. Then

$$
\lim _{n} \frac{1}{n^{2}} H_{n}^{(2)}=-\sup _{n} \frac{1}{n^{2}} H_{\pi_{n}}\left(\mu_{n}\right)-\int_{\mathbb{R}} f(t) \log f(t) d t
$$

Proof: With notation as in $\left(a_{0}\right)$, we can write $f\left(X_{n-1, n-1}^{n}\right)$ in the following form

$$
f\left(X_{n-1, n-1}^{n}\right)=\frac{f\left(X_{n-1, n-1}^{n}\right)}{\prod_{(s, t) \in I_{n-1, n-1}^{n}} f\left(x_{s, t}\right)} \times \prod_{(s, t) \in I_{n-1, n-1}^{n}} f\left(x_{s, t}\right),
$$

from which we get

$$
\int \log f\left(X_{n-1, n-1}^{n}\right) d \mu=\int \log \frac{f\left(X_{n-1, n-1}^{n}\right)}{\prod_{(s, t) \in I_{n-1, n-1}^{n}} f\left(x_{s, t}\right)} d \mu+\sum_{(s, t) \in I_{n-1, n-1}^{n}} \int \log f\left(x_{s, t}\right) f\left(x_{s, t}\right) d x_{s, t} .
$$

That is,

$$
\int \log f\left(X_{n-1, n-1}^{n}\right) d \mu=H_{\pi_{n}}\left(\mu_{n-1, n-1}^{n}\right)+n^{2} \int f\left(x_{0,0}\right) \log f\left(x_{0,0}\right) d x_{0,0} \quad \quad(* * * *)
$$

Put $z_{n}:=H_{\pi_{n}}\left(\mu_{n-1, n-1}^{n}\right)$. ( One can see that $\left(-z_{n}\right)$ is sub-additive.)

Let $R_{k, l}^{n}=\mathbb{Z}^{2} \cap[k n-1,(k+1) n-1] \times[l n-1,(l+1) n-1]$ and denote by $\mu \mid R_{k, l}^{n}$ the restriction of $\mu$ to the coordinates in $R_{k, l}^{n}$ and similarly for $\pi \mid R_{k, l}^{n}$. Let $n$ be fixed and $N \geq n$ be an integer. Write $N=p_{N} n+r_{N}=$ where, $p=p_{N}, r=r_{N} \in \mathbb{N}, 0 \leq r<n$ and $p \geq 1$. Then clearly we have, by the definition of the conditional entropy

$$
z_{N}=H_{\pi_{N}}\left(\mu_{N-1, N-1}^{N}\right) \geq H_{\pi_{p n}}\left(\mu_{p n-1, p n-1}^{p n}\right)=z_{p n} .
$$

But, by the Lemma 3.3 and invariance, we obtain

$$
z_{p n} \geq \sum_{k, l=0}^{p-1} H_{\pi \mid R_{k, l}^{n}}\left(\mu \mid R_{k, l}^{n}\right)=p^{2} H_{\pi \mid R_{0,0}^{n}}\left(\mu \mid R_{0,0}^{n}\right)=p^{2} z_{n}
$$


So

$$
\underset{N}{\limsup }\left[-\frac{1}{N^{2}} z_{N}\right] \leq-\frac{z_{n}}{n^{2}}
$$

which implies

$$
\limsup _{N}\left[-\frac{1}{N^{2}} z_{N}\right] \leq \inf _{n}\left[-\frac{z_{n}}{n^{2}}\right]
$$

As a consequence we can now define an entropy of absolutely continuous process indexed by $\mathbb{Z}^{2}$, as follows

\section{Definition 4.11:}

The Shannon entropy $S e(F, T, S)$ of the absolutely continuous process $\left(F \circ T^{m} \circ S^{n}\right)_{(m, n) \in \mathbb{Z}^{2}}$ is given by the equality

$$
S e(F, T, S):=\lim _{n}\left[-\frac{1}{n^{2}} \int \log f_{n^{2}} \circ F_{n^{2}} d \mu\right]=\lim _{n} \frac{1}{n^{2}} H_{n}^{(2)} .
$$

Note that here too it follows from Lemma 4.10 that $S e(F, T, S)=-\int f(t) \log f(t) d t$ if and only if the process $\left(F \circ T^{m} \circ S^{n}\right)_{(m, n) \in \mathbb{Z}^{2}}$ is independent.

In order to identify the limit in definition 4.11, for $F \in A C(\Omega, T, S, \mu)$, we need some further notation. Let

$$
g_{i, j}^{n}:=\frac{f\left(X_{i, j}^{n}\right)}{f\left(x_{0,0}\right) \times f\left(Y_{i, j}^{n}\right)}=\frac{d \mu_{i, j}^{n}}{d \nu_{i, j}^{n}},
$$

where $\mu, \nu$ are as in (21) and (20), respectively, and $\mu_{i, j}^{n}, \nu_{i, j}^{n}$ are as in $\left(r_{0}\right)$.

\section{Remark 4.12:}

With $\mu, \nu, g_{i, j}^{n}$ as in (21), (20) and (32), respectively, and $L$ as in $\left(a_{2}\right)$, the following properties hold: 
The family $\left(g_{i, j}^{n}\right)_{(i, j, n) \in L}$ is a $\nu$ martingale.

$$
\begin{gathered}
\int g_{i, j}^{n} \log g_{i, j}^{n} d \nu \leq \int g_{n-1, n-1}^{n} \log g_{n-1, n-1}^{n} d \nu \\
\sup _{(i, j, n) \in L} \int g_{i, j}^{n} \log g_{i, j}^{n} d \nu=\sup _{n} \int g_{n-1, n-1}^{n} \log g_{n-1, n-1}^{n} d \nu . \\
\frac{1}{n^{2}} h_{n}^{(2)}=-\frac{1}{n^{2}} \sum_{i, j=0}^{n-1} \log g_{i, j}^{n}-\log f\left(x_{0,0}\right) .
\end{gathered}
$$

\section{Remark 4.13}

Notations are as in Remark 4.12. Suppose $\sup _{n} \int g_{n-1, n-1}^{n} \log g_{n-1, n-1}^{n} d \nu<\infty$. Then if $s_{k}=$

$\left(i_{k}, j_{k}, n_{k}\right)$ is an increasing sequence in $L$, the martingale $M_{k}:=g_{i_{k}, j_{k}}^{n_{k}}$ is uniformly integrable and converges $\nu$ almost everywhere and in $L^{1}(\nu)$ to the density $g_{s}$ of $\mu$ restricted to $\sigma\left(\left\{M_{k}: k \geq 1\right\}\right)$ with respect to $\nu$.

In particular, if $U_{n}:=g_{n-1, n-1}^{n}$, then $U_{n}$ converges $\nu$ almost everywhere to the density $g$ of $\mu$ with respect to $\nu$ on the sigma-algebra $\sigma\left(\left\{x_{s, t}: s, t \geq 0\right\}\right)$ and therefore $g_{i, j}^{n}=E_{\nu}\left(g \mid \sigma\left(X_{i, j}^{n}\right)\right)$.

In fact, by (34), the hypothesis implies $\sup _{(i, j, n) \in L} \int g_{i, j}^{n}\left|\log g_{i, j}^{n}\right| d \nu<\infty$, so that the family $\left(g_{i, j}^{n}\right)_{(i, j, n) \in L}$ is uniformly integrable with respect to $\nu$. Here we used the following

\section{Remark 4.14:}

Let $m$ be a finite measure and $\Phi=\left\{f_{i}: i \in I\right\}$ be a family of positive elements in $L^{1}(m)$ with the property

$$
\sup _{i} \int f_{i} \log f_{i} d m<\infty
$$

Then (1) $\sup _{i} \int f_{i}\left|\log f_{i}\right| d m<\infty$.

(2) The family $\left\{f_{i}: i \in I\right\}$ is uniformly integrable.

(3) The family $\left\{\left(\log f_{i}\right)^{+}: i \in I\right\}$ is uniformly integrable.

The following lemma identifies the Shannon entropy of $\left(F \circ S^{m} \circ T^{n}\right)_{(m, n) \in \mathbb{Z}^{2}}$, for $\mathbb{Z}^{2}$ action (the 
limit in Lemma 4.10).

\section{Lemma 4.15}

Let $F \in A C(\Omega, T, S), \mu$ and $\nu$ be as in (21) and (20), respectively. Then

$$
S e(F, T, S)=\lim _{n} \frac{1}{n^{2}} H_{n}^{(2)}=-H_{\nu}(\mu)-\int f(t) \log f(t) d t
$$

and in particular $\operatorname{Se}(F, T, S)$ is finite if and only if $H_{\nu}(\mu)<\infty$.

Proof: Equation $(E)$ implies

$$
\frac{1}{n^{2}} H_{n}^{(2)}=-\frac{1}{n^{2}} \sum_{i, j=0}^{n-1} H_{\nu_{i, j}^{n}}\left(\mu_{i, j}^{n}\right)-\int f(t) \log f(t) d t,
$$

so

$$
\frac{1}{n^{2}} H_{n}^{(2)} \geq-H_{\nu_{n-1, n-1}^{n}}\left(\mu_{n-1, n-1}^{n}\right)-\int f(t) \log f(t) d t \geq-H_{\nu}(\mu)-\int f(t) \log f(t) d t,
$$

proving one direction. On the other hand, for every $n$, let $t_{n}=\int g_{n-1, n-1}^{n} \log g_{n-1, n-1}^{n} d \nu=$ $H_{\nu_{n-1, n-1}^{n}}\left(\mu_{n-1, n-1}^{n}\right)$, and $u_{n}=\frac{1}{n^{2}} \sum_{i, j=0}^{n-1} H_{\nu_{i, j}^{n}}\left(\mu_{i, j}^{n}\right)$. If $k>p$, then

$$
\begin{array}{r}
u_{k} \geq \frac{1}{k^{2}} \sum_{i=0}^{k-1} \sum_{j=p}^{k-1} H_{\nu_{i, j}^{k}}\left(\mu_{i, j}^{k}\right) \geq \frac{1}{k^{2}} \sum_{i=0}^{k-1} \sum_{j=p}^{k-1} H_{\nu_{p-1, p-1}^{p}}\left(\mu_{p-1, p-1}^{p}\right) \\
=\frac{k(k-p)}{k^{2}} H_{\nu_{p-1, p-1}^{p}}\left(\mu_{p-1, p-1}^{p}\right)=\left(1-\frac{p}{k}\right) H_{\nu_{p-1, p-1}^{p}}\left(\mu_{p-1, p-1}^{p}\right)=\left(1-\frac{p}{k}\right) t_{p} .
\end{array}
$$

Hence $\liminf _{k} u_{k} \geq t_{p}$, which implies $\liminf _{k} u_{k} \geq \sup _{p} t_{p}$, and proves the other direction and also the equality $H_{\nu}(\mu)=\lim _{n} u_{n}$, or that $\frac{1}{n^{2}} H_{n}^{(2)}$ converges to $-H_{\nu}(\mu)-\int f(t) \log f(t) d t$.

We give examples of non Gaussian and non independent process with finite Shannon entropy.

\section{Lemma 4.16:}

Let $(\Omega, \mu, T)$ be a dynamical system. Let $F, G \in L^{1}(\mu)$. Let $\xi_{n}:=\left(F, F \circ T, \ldots, F \circ T^{n-1}\right)$, and $\eta_{n}:=\left(G, G \circ T, \ldots, G \circ T^{n-1}\right)$. Suppose that $\xi_{n}$ is absolutely continuous and that $\xi_{n}$ and $\eta_{n}$ are independent. Then $\xi_{n}+\eta_{n}$ is absolutely continuous and

$$
H_{n}(F+G) \geq H_{n}(F)
$$


Proof: If $H_{n}(F)=-\infty$, there is nothing to prove. Suppose then that $H_{n}(F)>-\infty$. Denote by $f$ the density, with respect to $l^{n}$, of the law $\alpha$ of $\xi_{n}$, by $\mu$ the law of $\eta_{n}$, and by $\nu$ the law of $\xi_{n}+\eta_{n}$. Then $\nu=\alpha \star \mu=g d l^{n}$, and $g(t)=\int_{\mathbb{R}^{n}} f(t-y) d \mu(y)$, for $l^{n}$-almost all $t \in \mathbb{R}^{n}$. Thus we can write

$$
\begin{array}{r}
H_{n}(F+G)=\int_{\mathbb{R}^{n}} \psi(g) d l^{n}=\int_{\mathbb{R}^{n}} \psi\left(\int_{\mathbb{R}^{n}} f(t-y) d \mu(y)\right) d l^{n}(t) \\
\geq \int_{\mathbb{R}^{n}}\left[\int_{\mathbb{R}^{n}} \psi(f(t-y)) d \mu(y)\right] d l^{n}(t)=\int_{\mathbb{R}^{n}} d \mu(y) \int_{\mathbb{R}^{n}} \psi(f(u)) d l^{n}(u)=H_{n}(F) .
\end{array}
$$

\section{Corollary 4.17:}

Let $F \in A C(\Omega, T, \mu)$. Assume that $G \in L^{1}(\mu)$ is such that the two processes $\left(F \circ T^{n}\right)_{n \geq 0}$ and $\left(G \circ T^{n}\right)_{n \geq 0}$ are independent. Then

$$
S e(F+G, T) \geq S e(F, T) .
$$

If, in addition, $G \in A C(\Omega, T, \mu)$, then

$$
S e(F+G, T) \geq \max \{S e(F, T), S e(G, T)\} \geq \frac{1}{2}(S e(F, T)+S e(G, T)) .
$$

\subsection{Connections between Shannon entropy and Kolmogorov-Sinai entropy}

We show that if for some $F \in A C(\Omega, T)$, the Shannon entropy $S e(F, T)$ is finite then the Kolmogorv entropy of $(\Omega, T, \mu)$ is infinite (Corollary 4.21). We also give a new way to describe the Shannon entropy for $\mathbb{Z}$ action ( Theorem 4.20 (b)), and for $\mathbb{Z}^{2}$ action (Remark 4.22). Finally, we obtain a criterion of Markovianness (Proposition 4.23).

The following lemma is important for comparing the Kolmogorov-Sinai entropy to the Shannon entropy.

If $\mathcal{P}$ is a finite measurable partition of a probability space $(\Omega, \mathcal{F}, P)$ then the entropy of $\mathcal{P}$ will be denoted $H(\mathcal{P})$, or $H(\mathcal{P}, P)$ or $H^{P}(\mathcal{P})$. 


\section{Lemma 4.18:}

Let $\mathcal{E}$ be the set of finite measurable partitions of $\mathbb{R}$, and $F^{-1} \mathcal{E}=\left\{F^{-1} \mathcal{P}: \mathcal{P} \in \mathcal{E}\right\}$. Then

$$
H_{\mu F_{n}^{-1} \times \mu F_{p}^{-1}}\left(\mu F_{n+p}^{-1}\right)=\sup _{\mathcal{B} \in F^{-1} \mathcal{E}}\left[H^{\mu}\left(\bigvee_{j=0}^{n-1} T^{j} \mathcal{B}\right)-H^{\mu}\left(\bigvee_{j=0}^{n-1} T^{j} \mathcal{B} \mid \bigvee_{j=1}^{p} T^{-j} \mathcal{B}\right)\right]
$$

Proof: By Theorem B of Dobrushin,

$$
H_{\mu F_{n}^{-1} \times \mu F_{p}^{-1}}\left(\mu F_{n+p}^{-1}\right)=\sup S_{\mathcal{P}_{1} \times \mathcal{P}_{2}}\left(\mu F_{n+p}^{-1} \mid \mu F_{n}^{-1} \times \mu F_{p}^{-1}\right)
$$

the supremum being taken over all finite partitions $\mathcal{P}_{1}$ of $\mathbb{R}^{n}$ and $\mathcal{P}_{2}$ of $\mathbb{R}^{p}$, of the following particular forms: $\mathcal{P}_{1}=\mathcal{P} \times \ldots \times \mathcal{P}, n$ times, and $\mathcal{P}_{2}=\mathcal{P} \times \ldots \times \mathcal{P}, p$ times, where $\mathcal{P}$ run in $\mathcal{E}$. But it holds

$$
S_{\mathcal{P}_{1} \times \mathcal{P}_{2}}\left(\mu F_{n+p}^{-1} \mid \mu F_{n}^{-1} \times \mu F_{p}^{-1}\right)=-H\left(\mathcal{P}_{1} \times \mathcal{P}_{2}, \mu F_{n+p-1}^{-1}\right)+H\left(\mathcal{P}_{1}, \mu F_{n}^{-1}\right)+H\left(\mathcal{P}_{2}, \mu F_{p}^{-1}\right),
$$

and, for these particular forms of $\mathcal{P}_{i}, i=1,2$, setting $\mathcal{B}:=F^{-1} \mathcal{P}$, we easily have

$$
\begin{gathered}
H\left(\mathcal{P}_{1}, \mu F_{n}^{-1}\right)=H^{\mu}\left(\bigvee_{j=0}^{n-1} T^{-j} \mathcal{B}\right), \\
H\left(\mathcal{P}_{2}, \mu F_{p}^{-1}\right)=H^{\mu}\left(\bigvee_{j=n}^{n+p-1} T^{-j} \mathcal{B}\right), \text { and } H\left(\mathcal{P}_{1} \times \mathcal{P}_{2}, \mu F_{n+p-1}^{-1}\right)=H^{\mu}\left(\bigvee_{j=0}^{n+p-1} T^{-j} \mathcal{B}\right) .
\end{gathered}
$$

It follows then that

$$
S_{\mathcal{P}_{1} \times \mathcal{P}_{2}}\left(\mu F_{n+p}^{-1} \mid \mu F_{n}^{-1} \times \mu F_{p}^{-1}\right)=H^{\mu}\left(\bigvee_{j=0}^{n-1} T^{j} \mathcal{B}\right)-H^{\mu}\left(\bigvee_{j=0}^{n-1} T^{j} \mathcal{B} \mid \bigvee_{j=1}^{p} T^{-j} \mathcal{B}\right)
$$

In the light of lemma 4.18, formula (27) in Lemma 4.8 can be written as

$$
\sup _{\mathcal{B} \in F^{-1} \mathcal{E}}\left[H^{\mu}\left(\bigvee_{j=0}^{n-1} T^{j} \mathcal{B}\right)-H^{\mu}\left(\bigvee_{j=0}^{n-1} T^{j} \mathcal{B} \mid \bigvee_{j=1}^{p} T^{-j} \mathcal{B}\right)\right]=H_{n}+H_{p}-H_{n+p}
$$

\section{Remark 4.19:}

Formula (38) allows one to obtain conditions which ensure that $H_{n}$ will be finite.

One can prove for instance that, for all $N$, the following are equivalent: 
(i) $H_{N+1}$ is finite.

(ii) $\sup _{\mathcal{B} \in F^{-1} \mathcal{E}}\left[H^{\mu}(\mathcal{B})-H^{\mu}\left(\mathcal{B} \mid \bigvee_{j=1}^{N} T^{-j} \mathcal{B}\right)\right]<+\infty$

(ii') $H_{\mu F^{-1} \times \mu F_{N}^{-1}}\left(\mu F_{N+1}^{-1}\right)<+\infty$.

The following theorem gives, in particular, the announced new description of the Shannon entropy for $\mathbb{Z}$ action.

\section{Theorem 4.20:}

Let $\mathcal{E}$ be as in Lemma 4.18. Suppose $H_{n}$ finite for all $n$. Then

(a) The following are equivalent:

(i) $\operatorname{Se}(F, T)$ is finite.

(ii) For every $n$ (or for some $n$ ), $\sup _{\mathcal{B} \in F^{-1} \mathcal{E}}\left[H^{\mu}\left(\bigvee_{j=0}^{n-1} T^{j} \mathcal{B}\right)-n H^{\mu}\left(\mathcal{B} \mid \mathcal{B}^{-}\right)\right]<+\infty$.

(iii) $\lim _{n} \frac{1}{n} \sup _{\mathcal{B} \in F^{-1} \mathcal{E}}\left[H^{\mu}\left(\bigvee_{j=0}^{n-1} T^{j} \mathcal{B}\right)-n H^{\mu}\left(\mathcal{B} \mid \mathcal{B}^{-}\right)\right]=0$.

(b) The following equality holds

$$
\operatorname{Se}(F, T)=H_{1}+\inf _{\mathcal{B} \in F^{-1} \mathcal{E}}\left[H^{\mu}\left(\mathcal{B} \mid \mathcal{B}^{-}\right)-H^{\mu}(\mathcal{B})\right]
$$

Proof: Formulas (38), (27) and (28) imply the following

$$
z_{n}:=\sup _{\mathcal{B} \in F^{-1} \mathcal{E}}\left[H^{\mu}\left(\bigvee_{j=0}^{n-1} T^{j} \mathcal{B}\right)-H^{\mu}\left(\bigvee_{j=0}^{n-1} T^{j} \mathcal{B} \mid \mathcal{B}^{-}\right)\right]=H_{n}-n \times S e(F, T)
$$

in which as usual $\mathcal{B}^{-}:=T^{-1} \mathcal{B} \vee T^{-2} \mathcal{B} \vee \ldots$. Now

$$
H^{\mu}\left(\bigvee_{j=0}^{n-1} T^{j} \mathcal{B} \mid \mathcal{B}^{-}\right)=n H^{\mu}\left(\mathcal{B} \mid \mathcal{B}^{-}\right)
$$

so that formula (40) establishes the equivalence between $(i)$ and (ii). As $(i)$ implies (iii), by Remark 4.9, and trivially (iii) implies (ii), the proof is finished, because taking $n=1$ in formula (40) yields equality (39). 


\section{Corollary 4.21:}

Let $(\Omega, T, \mu)$ be an invertible dynamical system. If there exists $F \in A C(\Omega, T, \mu)$ such that the Shannon entropy $\operatorname{Se}(F, T)$ of the process $\left(F \circ T^{n}\right)$ is finite then $(\Omega, T, \mu)$ has infinite entropy.

Proof: The corollary follows directly from the equivalence between $(i)$ and $(i i)$ in Theorem 4.20.

Now, for $\mathbb{Z}^{2}$ action, we establish a formula analog to (39) for $\mathbb{Z}$ action.

\section{Remark 4.22:}

Let $\mathcal{E}$ be as in Lemma 4.18. Then, for the stationary absolutely continuous process $\left(F \circ S^{m} \circ T^{n}\right)$, indexed by $\mathbb{Z}^{2}$, the following holds

$$
S e(F, T, S)=-\int f(t) \log f(t) d t+\inf _{\mathcal{P} \in \mathcal{E}}\left[H^{\mu}\left(F^{-1} \mathcal{P} \mid\left(F^{-1} \mathcal{P}\right)^{-}\right)-H^{\mu}\left(F^{-1} \mathcal{P}\right)\right]
$$

In fact, we have $H_{\nu}(\mu)=\sup _{n} H_{\nu_{n}}\left(\mu_{n}\right)$. But

$$
H_{\nu_{n}}\left(\mu_{n}\right)=\sup S_{\Pi}\left(\mu_{n} \mid \nu_{n}\right)
$$

the supremum being taken over all partitions $\Pi$ of the form $\Pi=\left\{\cap_{(i, j) \in I_{n-1, n-1}^{n}} S^{-i} T^{-j} F^{-1} E: E \in\right.$ $\mathcal{P}$ \}, where $\mathcal{P}$ is a finite partition of $\mathbb{R}$. But then, with $E_{i, j}=S^{-i} T^{-j} F^{-1} E$, from the definitions of $\mu_{n}$ and $\nu_{n},($ see $(21)$ and (20)), it follows

$$
\begin{array}{r}
S_{\Pi}\left(\mu_{n} \mid \nu_{n}\right)=\sum_{E \in \mathcal{P}} \mu_{n}\left(\cap_{(i, j) \in I_{n-1, n-1}^{n}} E_{i, j}\right) \log \frac{\mu_{n}\left(\cap_{(i, j) \in I_{n-1, n-1}^{n}} E_{i, j}\right)}{\nu_{n}\left(\cap_{(i, j) \in I_{n-1, n-1}^{n}} E_{i, j}\right)}= \\
H^{\mu}\left(F^{-1} \mathcal{P}\right)-H^{\mu}\left(\bigvee_{(i, j) \in I_{n-1, n-1}^{n}} S^{-i} T^{-j} F^{-1} \mathcal{P}\right)+H^{\mu}\left(\bigvee_{(i, j) \in J_{n-1, n-1}^{n}} S^{-i} T^{-j} F^{-1} \mathcal{P}\right)= \\
H^{\mu}\left(F^{-1} \mathcal{P}\right)-H^{\mu}\left(F^{-1} \mathcal{P} \mid \bigvee_{(i, j) \in J_{n-1, n-1}^{n}} S^{-i} T^{-j} F^{-1} \mathcal{P}\right) .
\end{array}
$$


Hence

$$
\sup _{n} H_{\nu_{n}}\left(\mu_{n}\right)=\sup _{\mathcal{P}}\left[H^{\mu}\left(F^{-1} \mathcal{P}\right)-H^{\mu}\left(F^{-1} \mathcal{P} \mid\left(F^{-1} \mathcal{P}\right)^{-}\right)\right]
$$

where $\left.\left(F^{-1} \mathcal{P}\right)^{-}=\bigvee_{n \geq 1} \bigvee_{(i, j) \in J_{n-1, n-1}^{n}} T^{-i} S^{-j} F^{-1} \mathcal{P}\right)$

By Lemma 4.15, we thus obtain (41).

The following proposition gives a criterion for the Markoviannness of the process $\left(F \circ T^{n}\right)$.

\section{Proposition 4.23:}

Let $F \in A C(\Omega, T, m)$ such that $S e(F, T)$ is finite. Then

(i) The process $\left(F \circ T^{n}\right)$ is Markovian if and only if $S e(F, T)=H_{2}-H_{1}$. More generally

(ii) The process $\left(F \circ T^{n}\right)$ has memory $p$ if and only if $S e(F, T)=H_{p+1}-H_{p}$.

Proof: We prove $(i)$. The proof of $(i i)$ is similar. If $\left(F \circ T^{n}\right)$ is Markovian, we see by formulas (13) and (15), that $S e(F, T)=H_{2}-H_{1}$.

For the other direction, put, for $n \geq 1$,

$$
\left.\left.a_{n}:=\sup _{\mathcal{A} \in F^{-1} \mathcal{E}}\left[H(\mathcal{A})-H(\mathcal{A}) \mid T^{-1} \mathcal{A} \vee \ldots \vee T^{-n} \mathcal{A}\right)\right)\right]
$$

$\mathcal{R}^{n}=\mathcal{R}^{n}(\mathcal{A}):=T^{-2} \mathcal{A} \vee \ldots \vee T^{-n} \mathcal{A}$, and $L=L_{n}=E\left[. \mid T^{-1} \mathcal{A}\right]-E\left[. \mid T^{-1} \mathcal{A} \vee \mathcal{R}^{n}\right]$. Let $f$ be bounded $F^{-1} \mathcal{E}$-measurable function, where, as in Lemma $7, \mathcal{E}$ denotes the set of finite measurable partitions of $\mathbb{R}$. We shall show that $L(f)=0$ and the proof will be finished. To do this, we use a Lemma in [19], according to which for any $\epsilon>0$, there exists $\delta(\epsilon), 0<\delta(\epsilon)<\epsilon$, such that for any probability space $(\Omega, \mathcal{F}, \mu)$, and any finite partitions $\mathcal{P}$ and $\mathcal{Q}$ of $\Omega$, the inequality $H^{\mu}(\mathcal{P})-H^{\mu}(\mathcal{P} \mid \mathcal{Q})<\delta(\epsilon)$ implies that $\mathcal{P}$ and $\mathcal{Q}$ are $\epsilon$-inependent.

Recall that following Ornstein, if $\mathcal{P}$ and $\mathcal{Q}$ are finite measurable partitions of a probability space $(\Omega, \mathcal{F}, \mu)$, then $\mathcal{P}$ is said to be $\epsilon$-independent of $\mathcal{Q}$ if

$$
\sum_{p \in \mathcal{P}}|\mu(p \mid q)-\mu(p)|<\epsilon
$$


for all atom $q$ except a set of atoms of $\mathcal{Q}$ which union has a measure less than $\epsilon$.

Fix $\epsilon>0$. Then there exists $\mathcal{A}_{0}$ such that

$$
a_{1}-\delta(\epsilon)^{2}<H(\mathcal{A})-H\left(\mathcal{A} \mid T^{-1} \mathcal{A}\right)
$$

for any $\mathcal{A}$ which is finer than $\mathcal{A}_{0}$. But, by formula (15), the hypothesis $S e(F, T)=H_{2}-H_{1}$ is equivalent to $a_{1}=a_{n}$ for any $n$. Thus

$$
H\left(\mathcal{A} \mid T^{-1} \mathcal{A}\right)-H\left(\mathcal{A} \mid T^{-1} \mathcal{A} \vee \mathcal{R}^{n}(\mathcal{A})\right)<\delta(\epsilon)^{2} .
$$

So if we denote, respectively, by $p, q$ and $r$, the generic element of $\mathcal{A}, T^{-1} \mathcal{A}$ and $\mathcal{R}^{n}$, we get

$$
\sum_{q} m(q)\left[H^{m_{q}}\left(\mathcal{A}_{q}\right)-H^{m_{q}}\left(\mathcal{A}_{q} \mid \mathcal{R}_{q}^{n}\right)\right]<\delta(\epsilon)^{2}
$$

where $m_{q}(A)=\frac{m(A \cap q)}{m(q)}, \quad \mathcal{A}_{q}=\{p \cap q: p \in \mathcal{A}\}$ and similarly for $\mathcal{R}_{q}^{n}$.

Let $\mathcal{Q}_{\epsilon}:=\left\{q: H^{m_{q}}\left(\mathcal{A}_{q}\right)-H^{m_{q}}\left(\mathcal{A}_{q} \mid \mathcal{R}_{q}^{n}\right) \geq \delta(\epsilon)\right\}$. It follows that

$$
\left.\sum_{q \in \mathcal{Q}_{\epsilon}} m(q)\right)<\delta(\epsilon), \quad\left(e_{1}\right)
$$

and that, for $q \notin \mathcal{Q}_{\epsilon}$, the partitions $\mathcal{A}_{q}$ and $\mathcal{R}_{q}^{n}$ are $\epsilon$-independent, under the measure $m_{q}$, that is, there is $J_{q}$, a subfamily of $\mathcal{R}_{q}^{n}$, such that

$$
\sum_{r \in J_{q}} m_{q}(r)>1-\epsilon
$$

and

$$
\sum_{p \in \mathcal{A}}|m(p \mid q \cap r)-m(p \mid q)|<\epsilon, \quad \forall r \in J_{q} \quad \quad\left(e_{3}\right)
$$

Now we can find $\mathcal{A}$ finer than $\mathcal{A}_{0}$, and $g=g_{\epsilon}=\sum_{p \in \mathcal{A}} y_{p} 1_{p}$ such that $\|f-g\|_{1}<\epsilon$, and $\|g\|_{\infty} \leq 2 \times\|f\|_{\infty}$. Then 


$$
\|L(g)\|_{1}=\sum_{q, r}\left|\sum_{p} y_{p}[m(p \mid q)-m(p \mid q \cap r)]\right| m(q \cap r)=\sum_{q \in \mathcal{Q}_{\epsilon}}+\sum_{q \notin \mathcal{Q}_{\epsilon}} .
$$

In view of $\left(e_{1}\right)$, the first sum in the above equality, is bounded by $2\|g\|_{\infty} \delta(\epsilon)$. By $\left(e_{3}\right)$ and $\left(e_{2}\right)$, the second one is bounded by $3\|g\|_{\infty} \times \epsilon$.

Therefore

$$
\|L(g)\|_{1} \leq 2\|f\|_{\infty}[2 \delta(\epsilon)+3 \epsilon]
$$

It follows

$$
\|L(f)\|_{1} \leq 2\|f\|_{\infty}[2 \delta(\epsilon)+3 \epsilon]+2 \epsilon
$$

This implies $L(f)=0$.

Note that if $\left(F \circ T^{n}\right)$ is Markovian then for any $p, H_{2 p+1}=H_{1}+2 p\left(H_{2}-H_{1}\right)$ and $H_{2 p+2}=$ $H_{2}+2 p\left(H_{2}-H_{1}\right)$.

One might be tempted to introduce an isomorphism invariant $S e(T)$ :

$$
S e(T):=\sup _{F \in A C(\Omega, T \mu)}\left\{S e(F, T)-H_{1}(F, T)\right\}
$$

It is indeed an invariant, however, it can only take on the 2 values $-\infty$ and 0 .

\section{$5 \quad$ Entropy rate and Shannon entropy}

We shall establish some connections between Shannon entropy and some concepts developped by Pinsker such as information stability and entropy rate.

We recall some definitions from [14]. First recall that if $Z$ is a random variable then its law is 
denoted $P_{Z}$.

\section{Definition 5.1:}

Let $\xi=\left(\xi_{n}\right)_{n \geq 1}$ and $\eta=\left(\eta_{n}\right)_{n \geq 1}$ be discrete time stationary processes.

The entropy rate of $\xi$ with respect to $\eta$ is

$$
\bar{H}_{\eta}(\xi):=\lim _{n} \frac{1}{n} H_{P_{\left(\eta_{1}, \ldots, \eta_{n}\right)}}\left(P_{\left(\xi_{1}, \ldots, \xi_{n}\right)}\right),
$$

defined when, for all $j, \xi_{j}$ and $\eta_{j}$ take values in the same measurable space, and when the limit exists.

\section{Remark 5.2:}

We can prove, using Lemma 1, that if $\eta$ is the independent Gaussian process, then for any discrete time real state stationary process $\xi$, the entropy rate of $\xi$ with respect to $\eta$ is well defined.

\section{Lemma 5.3:}

Let $\eta=\left(X_{n}\right)$ be the independent gaussian process, with law $\gamma$ and $\gamma_{n}$ its projection on the first $n$ coordinates. Let $(\Omega, T, \mu)$ be a dynamical system, $F \in A C(\Omega, T, \mu)$ and $\nu$ the law of the process $\xi:=(F, F \circ T, \ldots)$. Then

(i) $S e(F, T)$ is finite if and only if $\sup _{n} \frac{1}{n} H_{\gamma_{n}}\left(\mu F_{n}^{-1}\right)<\infty$.

(ii) $\nu=\gamma$ if and only if $\lim _{n} \frac{1}{n} H_{\gamma_{n}}\left(\mu F_{n}^{-1}\right)=0$;

(iii) If $H_{\gamma}(\nu)$ is finite then $\nu=\gamma$.

Proof: Formula (23) implies

$$
S e(F, T)=\frac{1}{2}\left(\log (2 \pi)+\|F\|_{2}^{2}\right)-\lim _{n} \frac{1}{n} H_{\gamma_{n}}\left(\mu F_{n}^{-1}\right),
$$

from which we see that $S e(F, T)$ is finite if and only if $\lim _{n} \frac{1}{n} H_{\gamma_{n}}\left(\mu F_{n}^{-1}\right)$ is finite. This proves (i) because by super-additivity

$$
\lim _{n} \frac{1}{n} H_{\gamma_{n}}\left(\mu F_{n}^{-1}\right)=\sup _{n} \frac{1}{n} H_{\gamma_{n}}\left(\mu F_{n}^{-1}\right)
$$


Also this last equality together with Remark 3.2 (2) proves (ii).

To prove (iii) note that $H_{\gamma_{n}}\left(\mu F_{n}^{-1}\right) \leq H_{\gamma}(\nu)$, and formula (23) implies thus the inequality

$$
\frac{1}{n} H_{n}(F) \geq \frac{1}{2}\left(\log (2 \pi)+\|F\|_{2}^{2}\right)-\frac{1}{n} H_{\gamma}(\nu)
$$

which in turn implies the following one

$$
S e(F, T) \geq \frac{1}{2}\left(\log (2 \pi)+\|F\|_{2}^{2}\right)
$$

Hence, by corollary 4.5, we have $\nu=\gamma$

\section{Corollary 5.4:}

Let notations be exactly as in Lemma 5.3. Then

(i) $\operatorname{Se}(F, T)$ is finite if and only if the entropy rate of $\xi$ with respect to $\eta$ is finite.

(ii) $\nu=\gamma$ if and only if the entropy rate of $\xi$ with respect to $\eta$ vanishes.

(iii)

$$
\operatorname{Se}(F, T)=\frac{1}{2}\left(\log (2 \pi)+\|F\|_{2}^{2}\right)-\bar{H}_{\eta}(\xi) .
$$

\section{Lemma 5.5:}

Let $(\Omega, T, \mu)$ be a dynamical system, $F \in A C(\Omega, T, \mu)$ and $\nu$ the law of the process $\xi:=(F, F \circ T, \ldots)$. Let $P$ be the prduct measure $P:=\mu F^{-1} \otimes \mu F^{-1} \otimes \ldots$ and $P(n)=\left(\mu F^{-1}\right)^{\otimes n}$ its projection to the first $n$ coordinates. Then

(i) If for all $n \in \mathbb{N}, H_{n}$ is finite (in particular if $S e(F, T)$ is finite) $\nu$ is locally absolutely continuous with respect to $P: \forall n, \mu F_{n}^{-1}<<\left(\mu F^{-1}\right)^{\otimes n}$.

(ii) $S e(F, T)$ is finite if and only if $\sup _{n} \frac{1}{n} H_{\left(\mu F^{-1}\right) \otimes n}\left(\mu F_{n}^{-1}\right)$ is finite. 
(iii) $\nu=P \Longleftrightarrow \lim _{n} \frac{1}{n} H_{\left(\mu F^{-1}\right) \otimes n}\left(\mu F_{n}^{-1}\right)=0 \Longleftrightarrow S e(F, T)=\int_{\mathbb{R}} \psi\left(\frac{d \mu F^{-1}}{d l}\right) d l$.

(iv) If $H_{P}(\nu)$ is finite then $\nu=P$.

$(v)$

$$
S e(F, T)=\int_{\mathbb{R}} \psi\left(\frac{d \mu F^{-1}}{d l}\right) d l-\sup _{n} \frac{1}{n} H_{\left(\mu F^{-1}\right) \otimes n}\left(\mu F_{n}^{-1}\right) .
$$

Proof: Recalling that $\mathcal{I}_{n}(F)$ and $\mathcal{I}_{n, P M}(F)$ are defined respectively by (10) and (15), we have the following formula

$$
\mathcal{I}_{n}(F)=\mathcal{I}_{n, P M}(F)-\sum_{j=0}^{n-1} \log \frac{d \mu F^{-1}}{d l} \circ F \circ T^{j},
$$

which can be written as

$$
-\frac{1}{n} \log \frac{d \mu F_{n}^{-1}}{d l^{n}} \circ F_{n}(x)=-\frac{1}{n} \sum_{j=0}^{n-1} \log \frac{d \mu F^{-1}}{d l} \circ F \circ T^{j}(x)-\frac{1}{n} \log \frac{d \mu F_{n}^{-1}}{d\left(\mu F^{-1}\right)^{\otimes n}}\left(F_{n}(x)\right),
$$

a proof of which is as follows.

First, by taking in formula (7) in Lemma 3.3, $P_{1}=\left(\mu F^{-1}\right)^{\otimes n}$ and $P_{2}=\mu F^{-1}$, we find

$$
\begin{array}{r}
H_{\left(\mu F^{-1}\right) \otimes n \times \mu F^{-1}}\left(\mu F_{n+1}^{-1}\right)=H_{\mu F_{n}^{-1} \times \mu F^{-1}}\left(\mu F_{n+1}^{-1}\right)+H_{\left(\mu F^{-1}\right) \otimes n}\left(\mu F_{n}^{-1}\right) \\
+H_{\mu F^{-1}}\left(\mu F^{-1}\right)=H_{\mu F_{n}^{-1} \times \mu F^{-1}}\left(\mu F_{n+1}^{-1}\right)+H_{\left(\mu F^{-1}\right) \otimes n}\left(\mu F_{n}^{-1}\right) .
\end{array}
$$

And next, by taking $p=1$ in formula (27), we find

$$
H_{\left(\mu F^{-1}\right) \otimes n+1}\left(\mu F_{n+1}^{-1}\right)=H_{n}+H_{1}-H_{n+1}+H_{\left(\mu F^{-1}\right)^{\otimes n}}\left(\mu F_{n}^{-1}\right),
$$

from which it follows, when $H_{n+1}$ is finite, that $H_{\left(\mu F^{-1}\right) \otimes n+1}\left(\mu F_{n+1}^{-1}\right)$ is finite if and only if $H_{\left(\mu F^{-1}\right) \otimes n}\left(\mu F_{n}^{-1}\right)$ is finite. This, using Theorem A, implies, by induction on $n$, that if $H_{m}$ is finite for all $m$, then for every $n, \mu F_{n}^{-1}$ is absolutely continuous with respect to $\left(\mu F^{-1}\right)^{\otimes n}$. Thus 
we can write

$$
\frac{d \mu F_{n}^{-1}}{d l^{n}}=\frac{d \mu F_{n}^{-1}}{d\left(\mu F^{-1}\right)^{\otimes n}} \times \frac{d\left(\mu F^{-1}\right)^{\otimes n}}{d l^{n}}
$$

So, by taking logarithms, we obtain formula (46), from which follow immediately the formula (45), (ii), (iii) and (iv).

\section{Remark 5.6:}

Formula (46) implies

$$
H_{n}=H_{n, P M}+n \times\left[\frac{1}{2} \log (2 \pi)+\frac{1}{2}\|F\|_{2}^{2}-H_{\gamma_{0}}\left(\mu F^{-1}\right)\right] .
$$

In the same way we have

$$
\mathcal{I}_{n}(F)=\mathcal{I}_{n, G}(F)+\frac{n}{2} \log (2 \pi)+\frac{1}{2} \sum_{j=0}^{n-1} F^{2} \circ T^{j}
$$

and therefore (cf. formulas (22) and (23))

$$
H_{n}=H_{n, G}+n \times\left[\frac{1}{2} \log (2 \pi)+\frac{1}{2}\|F\|_{2}^{2}\right] .
$$

As a corollary, we obtain the following criterion for independence:

\section{Corollary 5.7:}

$\operatorname{Se}(F, T)=H_{1}(F)$ if and only if the process $\left(F \circ T^{n}\right)$ is independent.

Note that Corollary 5.7 can also be proved by using formula (7) and (23).

Corollary 5.7 together with formula (23) give the following improvement of Corollary 4.5 


\section{Corollary 5.8:}

Let $\gamma_{0}$ be the probability measure with density $\frac{1}{(2 \pi)^{\frac{1}{2}}} \exp \left(-\frac{1}{2} x^{2}\right)$ with respect to Lebesgue measure $l$ on $\mathbb{R}$. Let $F \in A C(\Omega, T, \mu)$. Then

$$
S e(F, T) \leq-H_{\gamma_{0}}\left(\mu F^{-1}\right)+\frac{1}{2}\left(\log (2 \pi)+\|F\|_{2}^{2}\right) .
$$

and the equality

$$
S e(F, T)=-H_{\gamma_{0}}\left(\mu F^{-1}\right)+\frac{1}{2}\left(\log (2 \pi)+\|F\|_{2}^{2}\right)
$$

holds if and only if the process $\left(F \circ T^{n}\right)$ is independent.

Note, once more, that we see from this corollary that $\operatorname{Se}(F, T)=\frac{1}{2}\left(\log (2 \pi)+\|F\|_{2}^{2}\right)$ if and only if the process $\left(F \circ T^{n}\right)$ is Gaussian independent [cf. Corollary 4.5].

We can also prove the following, which, in particular, improves the inequality (48) in the preceding corollary, and gives a link between the Shannon entropy and information stability.

\section{Lemma 5.9:}

If $\xi=\left(\xi_{n}\right)_{n \geq 1}$ and $\eta=\left(\eta_{n}\right)_{n \geq 1}$ are discrete time stationary processes, the rate of generation of information about $\eta$ by $\xi$ or about $\xi$ by $\eta$ (following Pinsker) is

$$
\bar{I}(\xi, \eta):=\lim _{n} \frac{1}{n} H_{P_{\left(\xi_{1}, \ldots, \xi_{n}\right)} \times P_{\left(\eta_{1}, \ldots, \eta_{n}\right)}}\left(P_{\left(\xi_{1}, \ldots, \xi_{n}\right),\left(\eta_{1}, \ldots, \eta_{n}\right)}\right) .
$$

The pair $(\xi, \eta)$ is called information stable if $\bar{I}(\xi, \eta)=0$.

Let $(\Omega, T, \mu)$ be an invertible dynamical system and $F \in A C(\Omega, T, \mu)$. Let $\phi$ and $\pi$ be the processes defined by $\phi_{n}=F \circ T^{-n+1}$, and $\pi_{n}=F \circ T^{n}$, for $n=1,2, \ldots$ Then

(i) The pair $(\phi, \pi)$ is information stable if and only if $\lim _{n}\left(\frac{H_{n}}{n}-\frac{H_{2 n}}{2 n}\right)=0$. In particular, if $S e(F, T)$ is finite the pair $(\phi, \pi)$ is information stable. 
(ii) $S e(F, T)$ is finite if and only if $\sum_{p=0}^{\infty} \frac{1}{2^{p}} H_{\mu F_{2^{p}}^{-1} \times \mu F_{2^{p}}^{-1}}\left(\mu F_{2^{p+1}}^{-1}\right)<\infty$. Moreover

(iii) $S e(F, T)=\frac{1}{2}\left(\log (2 \pi)+\|F\|_{2}^{2}\right)-H_{\gamma_{0}}\left(\mu F^{-1}\right)-\frac{1}{2} \sum_{p=0}^{\infty} \frac{1}{2^{p}} H_{\mu F_{2^{p}}^{-1} \times \mu F_{2^{p}}^{-1}}\left(\mu F_{2^{p+1}}^{-1}\right)$.

We also have

\section{Remark 5.10:}

Le $\Omega, T, \mu)$ be a dynamical system, $F \in A C(\Omega, T, \mu)$ and $\xi$ the process $\xi:=(F, F \circ T, \ldots)$. Then the entropy rate of $\xi$ with respect to the independent Gaussian stationary process $\eta$ is given by

$$
\bar{H}_{\eta}(\xi)=H_{\gamma_{0}}\left(\mu F^{-1}\right)+\frac{1}{2} \sum_{p=0}^{\infty} \frac{1}{2^{p}} H_{\mu F_{2^{p}}^{-1} \times \mu F_{2^{p}}^{-1}}\left(\mu F_{2^{p+1}}^{-1}\right)
$$

\section{Application to Gaussian processes}

In this section we express the Shannon entropy $S e(F, T)$ in terms of the spectral measure of the Gaussian process $X=\left(X_{n}\right)_{n \in \mathbb{Z}}$, when $F=X_{0}$ is the zero coordinate function ( Lemma 6.3 ). This enables us (1) to prove that in the class of Gaussian Markovian processes, the Shannon entropy almost determines the process ( Remark 6.4 ), (2) to show how this entropy changes by linear change of variable ( Corollary 6.5 ), and (3) to prove that all unilateral Gaussian processes with finite Shannon entropy are isomorphic ( Theorem 6.6 ).

We need first some preliminaries.

Let $\left(v_{n}\right)_{n \geq 0}$ be a stationary sequence of unit vectors in the real Hilbert space $H$, with $(r(n))_{n \in \mathbb{Z}}$ strictly positive definite sequence (defining $r(-n)=r(n)$ ), where $r(n)=<v_{n}, v_{0}>=<v_{n+k}, v_{k}>$. Let $R_{n}$ be the $n \times n$ matrix $\left(R_{n}\right)_{i j}=r(i-j)$, for $i, j=0, \ldots n-1$ and $r$ be the vector $r=$ 
$[r(1), \ldots, r(n-1)]^{t}$. Then

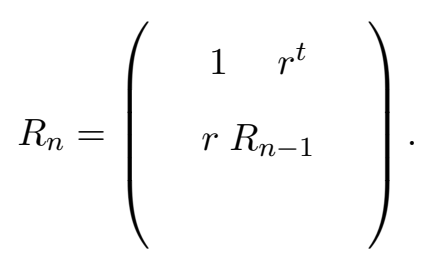

Evidently for each $n$ there exists a unique vector $a=\left[a_{1}, \ldots, a_{n}\right]^{t}$ such that the vector

$$
w_{n}:=v_{0}-\sum_{i=1}^{n-1} a_{i} v_{i}
$$

is orthogonal to $v_{i}$ for $i=1, \ldots, n-1$. Using the orthogonal decomposition

$$
v_{0}=w_{n}+\left(a_{1} v_{1}+\ldots+a_{n-1} v_{n-1}\right),
$$

we can prove, by taking scalar products $<v_{0}, v_{0}>, \ldots,<v_{n-1}, v_{0}>$, that $a$ is given by the equation $r=R_{n-1} a$, or

$$
a=R_{n-1}^{-1} r .
$$

\section{Lemma 6.1:}

For any $X^{t}=\left(x_{0}, \ldots, x_{n-1}\right)$ set $Y^{t}=\left(x_{1}, \ldots, x_{n-1}\right)$. Then we have

$$
X^{t} R_{n}^{-1} X-Y^{t} R_{n-1}^{-1} Y=\frac{\left(x_{0}-\sum_{j=1}^{n-1} a_{j} x_{j}\right)^{2}}{\left\|w_{n}\right\|^{2}}
$$

and

$$
\operatorname{det}\left(R_{n}\right)=\left\|w_{n}\right\|^{2} \operatorname{det}\left(R_{n-1}\right)
$$

In the same way, note first that we have the equality

$$
R_{n}=\left(\begin{array}{cc}
R_{n-1} & r^{i} \\
\left(r^{i}\right)^{t} & 1
\end{array}\right)
$$


where $r^{i}$ is the vector whose transpose is $\left(r^{i}\right)^{t}=(r(n-1), \ldots, r(1))$. And for any $n$ there exists a unique vector $b=\left[b_{0}, \ldots, b_{n-2}\right]^{t}$ such that the vector $u_{n}$ defined by

$$
u_{n}=v_{n-1}-b_{0} v_{0}-\ldots-b_{n-2} v_{n-2}
$$

is orthogonal to $v_{j}$ for $j=0, \ldots, n-2$. So $v_{n-1}-u_{n}$ is the projection of $v_{n-1}$ onto the subspace spanned by $v_{0}, \ldots, v_{n-2}$, and $u_{n}$ is the projection of $v_{n-1}$ onto the orthogonal of the linear span of $\left\{v_{0}, \ldots, v_{n-2}\right\}$. Since

$$
v_{n-1}=u_{n}+\left(b_{0} v_{0}+\ldots+b_{n-2} v_{n-2}\right)
$$

an "orthogonal decomposition", we obtain, by taking scalar products $<v_{0}, v_{n-1}>, \ldots,<v_{n-1}, v_{n-1}>$, the equality $r^{i}=R_{n-1} b$, or

$$
b=R_{n-1}^{-1} r^{i}
$$

We have also the following

\section{Lemma 6.2:}

If $X=\left[x_{0}, \ldots, x_{n-1}\right]^{t}$ and $Y=\left[x_{0}, \ldots, x_{n-2}\right]^{t}$, then

$$
X^{t} R_{n}^{-1} X-Y^{t} R_{n-1}^{-1} Y=\frac{\left(x_{n-1}-\sum_{j=0}^{n-2} b_{j} x_{j}\right)^{2}}{\left\|u_{n}\right\|^{2}}
$$

and

$$
\operatorname{det}\left(R_{n}\right)=\left\|u_{n}\right\|^{2} \operatorname{det}\left(R_{n-1}\right)
$$


Proof of Lemma 6.2: Let

$$
Q=\left(\begin{array}{cc}
I_{n-1} & 0 \\
-b^{t} & 1 \\
&
\end{array}\right)
$$

where $I_{n-1}$ is the identity matrix. Then

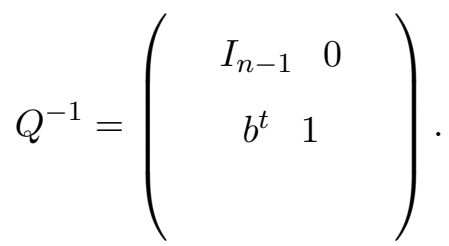

and we have

$$
\left(Q^{-1}\right)^{t} R_{n}^{-1} Q^{-1}=\left(\begin{array}{cc}
R_{n-1}^{-1} & 0 \\
0 \ldots 0 & \alpha \\
&
\end{array}\right)
$$

where $\alpha=\frac{1}{\left\|u_{n}\right\|^{2}}$. In fact this equality is equivalent to

$$
Q R_{n} Q^{t}=\left(\begin{array}{cc}
R_{n-1} & 0 \\
0 \ldots 0 & \alpha^{-1}
\end{array}\right)
$$

which can be easily verified.

Now let $\Omega=\mathbb{R}^{\mathbb{Z}}, \sigma$ the shift transformation, $\mu$ a Gaussian $\sigma$ invariant probability measure deter-

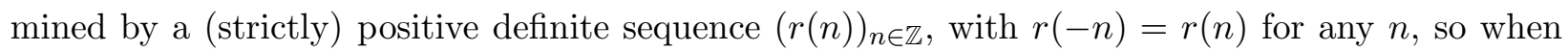
$r(0)=1$, there exists a probability measure $\nu$ on the unit circle $\mathbb{T}$ such that $\hat{\nu}(n)=r(n)$. In this case we shall call $\nu$ the spectral measure. In other words, each $n$ dimensional distribution has a density $\rho_{n}$ given by

$$
\rho_{n}\left(x_{0}, \ldots, x_{n-1}\right)=\frac{1}{(2 \pi)^{\frac{n}{2}}\left(\operatorname{det} R_{n}\right)^{\frac{1}{2}}} \times \exp \left(-\frac{1}{2} \sum_{i, j=0}^{n-1}\left(R_{n}^{-1}\right)_{i j} x_{i} x_{j}\right)
$$


where

$$
\left(R_{n}\right)_{i j}=r(i-j)=\int_{\Omega} x_{i} x_{j} d \mu(x), \quad i, j=0, \ldots, n-1
$$

So, if $F(x)=x_{0}$ for $x \in \Omega$, then, for $i \geq j$,

$$
\left(R_{n}\right)_{i j}=\int_{\Omega} F \circ \sigma^{i-j} F d \mu
$$

\section{Lemma 6.3:}

Let $P_{n}, Q_{n}$ and $Q$ denote the orthogonal projections onto the linear span of $\left\{X_{1}, \ldots, X_{n}\right\},\left\{X_{-n+1}, \ldots, X_{-1}\right\}$ and $\left\{X_{-1}, X_{-2}, \ldots\right\}$ respectively. Then

a)

$$
S e(F, \sigma)=\frac{1}{2} \log (2 \pi)+\log \|F-Q F\|_{2}+\frac{1}{2}=\frac{1}{2} \log (2 \pi)+\frac{1}{2} \int \log f d \lambda+\frac{1}{2} . \quad(* *)
$$

where $f$ is the density of $\nu$ with respect to $\lambda$.

In particular, if $\|F-Q F\|=0$ then $\operatorname{Se}(F, \sigma)=-\infty$.

b) If $\|F-Q F\|>0$, the following are equivalent

(i) The Shannon information $\frac{1}{n} \mathcal{I}_{n}(F)$ converges almost everywhere [resp. in $\left.L^{1}\right]$.

(ii) $\frac{1}{N} \sum_{j=1}^{N-1}\left(F-P_{j} F\right)^{2}\left(\sigma^{N-j}\right)$ converges almost eveywhere [resp. in $\left.L^{1}\right]$.

(iii) $\frac{1}{N} \sum_{j=1}^{N-1}\left(F-P_{j} F\right)^{2}\left(\sigma^{-j}\right)$ converges almost eveywhere [resp. in $\left.L^{1}\right]$.

(iv) $\frac{1}{N} \sum_{j=1}^{N-1}\left(F-Q_{j} F\right)^{2}\left(\sigma^{j}\right)$ converges almost eveywhere [resp. in $L^{1}$ ].

(v) $\frac{1}{N} \sum_{n=2}^{N} \log \frac{\rho_{n}(x)}{\rho_{n}(\sigma x)}$ converges almost everywhere [resp. in $\left.L^{1}\right]$.

Proof: By formula (54) and lemma 6.1 we get

$$
-\log \frac{\rho_{n}\left(x_{0}, \ldots, x_{n-1}\right)}{\rho_{n-1}\left(x_{1}, \ldots, x_{n-1}\right)}=\frac{1}{2} \log (2 \pi)+\log \left\|F-P_{n-1} F\right\|_{2}+\frac{1}{2} \frac{\left(F-P_{n-1} F\right)^{2}(x)}{\left\|F-P_{n-1} F\right\|_{2}^{2}}
$$

Now, since the process is Gaussian, $P_{n-1} F$ converges to $P F$ almost everywhere and in $L^{2}$, where $P$ is the projection onto the linear span of $\left\{F \circ \sigma, F \circ \sigma^{2}, \ldots\right\}$, and $P F=E\left(F \mid \sigma^{-1} \mathcal{B}\right)$, where $\mathcal{B}$ is 
the Borel sigma-algebra. So, in the case where $\|F-P F\|_{2}>0$, it follows from (56), that

$$
\lim _{n}\left[-\log \frac{\rho_{n}(x)}{\rho_{n-1}(\sigma x)}\right]=\frac{1}{2} \log (2 \pi)+\log \|F-P F\|_{2}+\frac{1}{2} \frac{(F-P F)^{2}(x)}{\|F-P F\|_{2}^{2}}
$$

Then the equality

$$
-\frac{1}{N} \sum_{n=3}^{N} \log \frac{\rho_{n}(x)}{\rho_{n-1}(\sigma x)}=-\frac{1}{N} \sum_{n=3}^{N-1} \log \left(\frac{\rho_{n}(x)}{\rho_{n}(\sigma x)}\right)-\frac{1}{N} \log \rho_{N}(x)+\frac{1}{N} \log \rho_{2}(\sigma x)
$$

proves that $-\frac{1}{N} \log \rho_{N}(x)$ converges almost surely [ respectively in $L^{1}$ ] if and only if $-\frac{1}{N} \sum_{n=3}^{N-1} \log \left(\frac{\rho_{n}(x)}{\rho_{n}(\sigma x)}\right)$ converges almost surely [respectively in $\left.L^{1}\right]$. Now, by (56) and (58), we obtain the equality $(* *)$. In the same way, we get, by Lemma 6.2

$$
-\log \frac{\rho_{n}\left(x_{0}, \ldots, x_{n-1}\right)}{\rho_{n-1}\left(x_{0}, \ldots, x_{n-2}\right)}=\frac{1}{2} \log (2 \pi)+\frac{1}{2} \log \frac{\operatorname{det} R_{n}}{\operatorname{det} R_{n-1}}+\frac{1}{2} \frac{\left(x_{n-1}-\sum_{j=0}^{n-2} b_{j} x_{j}\right)^{2}}{\left\|u_{n}\right\|^{2}} .
$$

But, if $L_{n-2}$ is the orthogonal projection onto the linear span of $X_{0}, \ldots, X_{n-2}$, we have $\left\|u_{n}\right\|^{2}=\|$ $X_{n-1}-L_{n-2} X_{n-1}\left\|_{2}^{2}=\right\| F-Q_{n} F \|_{2}^{2}$, and thus

$$
\begin{gathered}
\frac{1}{N-1}\left[-\log \rho_{N}\left(x_{0}, \ldots, x_{N-1}\right)+\log \rho_{1}\left(x_{0}\right)\right]=\frac{1}{2} \log (2 \pi)+\frac{1}{N-1} \sum_{n=2}^{N} \log \left\|F-Q_{n} F\right\|_{2} \\
+\frac{1}{2(N-1)} \sum_{n=2}^{N} \frac{\left(F-Q_{n} F\right)^{2} \circ \sigma^{n-1}(x)}{\left\|F-Q_{n} F\right\|_{2}^{2}} . \quad(* * *)
\end{gathered}
$$

Then, in the case where $\|F-Q F\|>0$, the sequence of Shannon informations $\frac{1}{n} \mathcal{I}_{n}(F)$ converges a.e. [ respectively in $L^{1}$ ] if and only if $\frac{1}{N} \sum_{j=1}^{N-1}\left(F-Q_{j} F\right)^{2}\left(\sigma^{j}\right)$ does so.

The other statements can be proved in a similar way.

We can see easily from $(* *)$ the following

\section{Remark 6.4:}

Let $\left(X_{n}\right)$ and $\left(Y_{n}\right)$ be stationary centered Gaussian Markovian processes, with the same $L^{2}$ norm. Then they have the same Shannon entropy if and only if either they have the same law, or $\left(Y_{n}\right)$ 
and $\left((-1)^{n} X_{n}\right)$ have the same law.

Proof: Let $\nu$ be the spectral measure of $X$ and $\nu^{\prime}$ be the spectral measure of $Y$. Then if $P_{r}(t)=\sum_{n \in \mathbb{Z}} r^{|n|} e^{i n t}$ is the Poisson kernel, we have $\nu=P_{r}(t) d \lambda(t)$, for some $r$ and similarly $\nu^{\prime}=P_{r^{\prime}}(t) d \lambda(t)$, for some $r^{\prime}$. On the other hand, form $(* *)$, the equality $\operatorname{Se}\left(X_{0}, \sigma\right)=\operatorname{Se}\left(Y_{0}, \sigma\right)$ holds if and only if $\left\|X_{0}-Q X_{0}\right\|=\left\|Y_{0}-Q^{\prime} Y_{0}\right\|$, where $Q^{\prime}$ denotes the projection to the negative coordinates of $Y$. But $Q X_{0}=a X_{-1}$ and similarly $Q^{\prime} Y_{0}=b Y_{-1}$, for some constants $a, b$. Thus the equality of the respective Shannon entropies is equivalent to $|a|=|b|$, or to $a<X_{-1}, X_{0}>=b<Y_{-1}, Y_{0}>$, that is to $a r=b r^{\prime}$. [ Also, one can show by elementary calculus that $\int_{\mathbb{T}} \log P_{r}(t) d \lambda(t)=\int_{\mathbb{T}} \log P_{r^{\prime}}(t) d \lambda(t)$ if and only if $\left.|r|=\left|r^{\prime}\right|.\right]$

\section{Corollary 6.5:}

Let $\left(X_{n}\right)_{n \in \mathbb{Z}}$ be Gaussian stationary process with spectral measure $\nu$. Let $g=\sum_{n \in \mathbb{Z}} a_{n} e^{i n t} \in L^{2}(\nu)$ and $\left(Y_{n}\right)_{n \in \mathbb{Z}}$ be the stationary Gaussian process such that $Y_{0}=\sum_{n \in \mathbb{Z}} a_{n} X_{n}$. Then

$$
S e\left(Y_{0}, \sigma\right)=S e\left(X_{0}, \sigma\right)+\int_{\mathbb{T}} \log (|g|) d \lambda
$$

Proof: By the spectral theorem, if $\nu^{\prime}$ is the spectral measure of $\left(Y_{n}\right)$, we have $\nu^{\prime}=|g|^{2} \nu$. So, by Szego Theorem

$$
\left\|Y_{0}-Q^{\prime} Y_{0}\right\|^{2}=\exp \left[\int \log \left(|g|^{2} \frac{d \nu}{d \lambda}\right) d \lambda\right]=\exp \left[\int \log \left(|g|^{2}\right) d \lambda\right]\left\|X_{0}-Q X_{0}\right\|^{2}
$$

Thus, by $(* *)$, we get the result.

Note that, if $g \in L^{1}(\lambda)$, then $\int_{\mathbb{T}} \log (|g|) d \lambda$ is finite if and only if there is $h \in H^{1}$ such that $|g|=|h|$, and in this case, $\int \log (|g|) d \lambda \geq|h(0)|=\left|\int h d \lambda\right|$.

In particular if $a_{n}=0$ for $n>0$ (or for $n<0$ ) and $g \in L^{1}(\lambda) \cap L^{2}(\nu)$ then $\int \log (|g|) d \lambda \geq \log \mid$ $\int g d \lambda|=\log | a_{0} \mid$. 
More particularly, if $g \in \mathbb{H}^{1}(\mathbb{T})$ is an outer function, then $\int \log (|g|) d \lambda=\log \left|\int g d \lambda\right|$, and when, in addition $\int g d \lambda=0$, we obtain $S e\left(Y_{0}, \sigma\right)=S e\left(X_{0}, \sigma\right)$.

It is well known from Ornstein theory that a bilateral gaussian process $X=\left(X_{n}\right)_{n \in \mathbb{Z}}$ with spectral measure absolutely continuous with respect to Lebesgue measure on the circle is isomorphic to the gaussian independent process. There is interest in considering isomorphism for non-invertible transformations (endomorphisms). The first examples of such isomorphism has been worked out by Parry [13] and elaborated by Hoffman and Rudolph [6] ( All the endomorphisms they consider are finite to one.). We consider now the unilateral transformation (endomorphism) associated to gaussian process with spectral measure equivalent to Lebesgue measure $\lambda$. For clarity, if $X=$ $\left(X_{n}\right)_{n \geq 0}$ is a Gaussian process with spectral measure $\nu=f d \lambda$ we consider the endomorphism $T_{\nu}$ defined on $\mathbb{R}^{\mathbb{N}}$ by $\left(T_{\nu} x\right)_{n}=x_{n+1}$, for $x \in \mathbb{R}^{\mathbb{N}}$ and $n \geq 0$. Then the shift $T_{\nu}$ will be isomorphic to the shift $T_{\lambda}$ if and only if $\log f$ is Lebesgue integrable.

To prove this we recall some useful properties that functions in $\mathbb{H}^{1}$ or in $\mathbb{H}^{2}$ can have. First recall that, for $p=1,2, \mathbb{H}^{p}$ is the closed subspace of all $f \in L^{p}\left(\mathbb{T}, \frac{d \theta}{2 \pi}\right)$ such that $\int_{-\pi}^{\pi} f(t) e^{i n t} d t=0, n=$ $1,2 \ldots$, and that if $0 \leq f \in L^{1}$ then $\log f$ is integrable if and only if there is $F \in \mathbb{H}^{2}$ such that $f=|F|^{2}$ [ [5], Theorem, p.53]. Recall also that an inner function $f$ is an analytic function in the unit disc such that $|f(z)| \leq 1$ and $\left|f\left(e^{i \theta}\right)\right|=1$ almost everywhere on the unit circle, and an outer function $F$ is an an analytic function in the unit disc of the form

$$
F(z)=\alpha \exp \left[\frac{1}{2 \pi} \int_{-\pi}^{\pi} \frac{e^{i \theta}+z}{e^{i \theta}-z} k(\theta) d \theta\right]
$$

where $k$ is a real-valued integrable function on the circle and $\alpha$ is a complex number with modulus $1\left[[5]\right.$, p.63,]. For a function $F \in \mathbb{H}^{2}$ to be an outer function it is necessary and sufficient that the family $\left\{z^{n} F: n=0,1 \ldots\right\}$ span $\mathbb{H}^{2}\left[[5]\right.$, corollary, p. 101]. Also any non zero function $f \in \mathbb{H}^{1}$ can be written in the form $f=g F$ where $g$ is inner and $F$ is outer [ [5], Theorem, p. 63, [4], Theorem $12]$.

In the next theorem the use of Shannon entropy is only to ensure that the logarithm of the density of the spectral measure is integrable. 


\section{Theorem 6.6:}

Let $\nu$ be a probability measure on the unit circle, equivalent to Lebesgue measure $\lambda$, with density $f$. Then the unilateral shifts $T_{\nu}$ and $T_{\lambda}$ are isomorphic if and only if $\operatorname{Se}\left(X_{0}, T_{\nu}\right)$ is finite, or equivalently $\log f$ is Lebesgue integrable.

Proof: Consider the two bilateral gaussian processes $X^{\prime}$ and $Y^{\prime}$ with spectral measures $\lambda$ and $f d \lambda$ respectively. Then on the cyclic space $Z_{X_{0}}=\left\{X_{0} \circ T^{n}: n \in \mathbb{Z}\right\}, T$ is unitarily equivalent to the multiplication $M_{z}$ by $z$ on $L^{2}(\lambda)$. An isomorphism $\phi$ is given by $\phi\left(X_{n}\right)=z^{n}, n \in \mathbb{Z}$. Similarily, the same holds for $T$ on the cyclic space $Z_{Y_{0}}=\left\{Y_{0} \circ T^{n}: n \in \mathbb{Z}\right\}$ and the multiplication by $z$ on $L^{2}(f d \lambda)$, with isomorphism $\psi: \psi\left(Y_{n}\right)=z^{n}, n \in \mathbb{Z}$. It follows that the action of $T$ on $Z_{X_{0}}$ is unitarily equivalent to the action of $T$ on $Z_{Y_{0}}$. Suppose first that $\log f$ is integrable. Then there exists $F \in \mathbb{H}^{2}$ such that $\sqrt{f}=|F|$. Moreover, there exist an inner function $g$ and an outer function $G \in \mathbb{H}^{2}$ such that $F=g G$ and thus $|F|=|G|$. Set $x=\phi^{-1} G$, so that $x$ belongs to the closed linear span of $\left\{X_{0}, X_{1}, \ldots\right\}$, and we have

$$
\begin{array}{r}
<T^{n} x, x>=<T^{n} \phi^{-1} G, \phi^{-1} G>=<\phi^{-1} M_{z}^{n} G, \phi^{-1} G> \\
=<M_{z}^{n} G, G>=\int z^{n}|G|^{2} d \lambda=\int z^{n} f d \lambda=<T^{n} Y_{0}, Y_{0}>.
\end{array}
$$

On the other hand, if $P=\sum_{k} a_{k} z^{k}$ is a polynomial, the following equalities

$$
\begin{aligned}
\| X_{0}-\sum_{k} a_{k} T^{k} x & \|=\| \phi\left(X_{0}\right)-\sum_{k} a_{k} \phi\left(T^{k} x\right)\|=\| 1-\sum_{k} a_{k} M_{z}^{k} \phi(x) \| \\
& =\left\|1-\sum_{k} a_{k} M_{z}^{k} G\right\|=\left\|1-\sum_{k} a_{k} z^{k} G\right\|=\|1-P G\|,
\end{aligned}
$$

prove that $X_{0}$ belongs to the closed linear space generated by $\left\{T^{n} x: n \geq 0\right\}$ if and only if 1 belongs to the closed linear space generated by $\left\{z^{n} G: n \geq 0\right\}$. But, since $G$ is outer, this later is equal to $\mathbb{H}^{2}$ and thus $X_{0} \in \overline{\operatorname{lin}}\left\{T^{n} x: n \geq 0\right\}$. This proves that $T_{\nu}$ and $T_{\lambda}$ are isomorphic. The other implication follows from Szegö Theorem. 
We end this section with the following result concerning the speed of convergence in linear prediction:

\section{Proposition 6.7:}

Let $\lambda$ be the Lebesgue probability measure on $\mathbb{T}$, and $\nu$ the spectral measure of a stationary Gaussian process $\left(X_{n}\right)_{n \in \mathbb{Z}}$. Let $Q$ denote the orthogonal projection onto the closed (in $L^{2}(\nu)$ ) linear span of the negative coordinates and $Q_{n}$ be the orthogonal projection onto the linear span of $\left\{X_{-n}, \ldots, X_{-1}\right\}$. Suppose that $X_{0} \neq Q X_{0}$, or equivalently $\log \frac{d \nu}{d \lambda}$ is Lebesgue integrable. Then:

The series $\sum_{n=1}^{\infty}\left\|Q X_{0}-Q_{n} X_{0}\right\|_{2}^{2}$ converges if and only if $\nu$ is absolutely continuous and $\nu=e^{f} d \lambda$, with $\sum_{n=1}^{\infty} n|\hat{f}(n)|^{2}<\infty$.

An equivalent form of Proposition 3 is

\section{Remark 6.8:}

Let $\nu$ be a probability measure on $\mathbb{T}$. Let $H, H_{n}$ denote the closed subspaces of $L^{2}(\nu)$ spanned by $\left\{e^{i k t}: k \geq 1\right\}$, and $\left\{e^{i k t}: 1 \leq k \leq n\right\}$ respectively. Let $F, F_{n}$ be the orthogonal projection of the constant function 1 onto $H$ and $H_{n}$ respectively. Suppose that 1 is not in $H$. Then the following are equivalent

(i) $\sum_{n=1}^{\infty}\left\|F-F_{n}\right\|^{2}<\infty$.

(ii) $\nu$ is absolutely continuous with respect to the Lebesgue probability measure $\lambda$ and $\nu=e^{f} d \lambda$, with $\sum_{n=1}^{\infty} n|\hat{f}(n)|^{2}<\infty$. 


\section{$7 \quad \mathbb{Z}^{2}$ action, pointwise statement}

In this section we consider specifically absolutely continuous $\mathbb{Z}^{n}$ processes, for which we prove pointwise convergence of the Shannon entropy. The case where $n=1$ has already been considered by Barron [1]. However his method can not extend to the higher dimensional case; the idea of our proof is very related to the one by Ornstein and Weiss [10] for the $\mathbb{Z}^{n}$ version of the Shannon Mac Millan Breiman Theorem. The proof is given for $n=2$, but it can be easily generalized.

Notations are as in sections 4. Particularly, we refer to (16), (18) for $h_{n}^{(2)}$ and to Definition 4.11, Lemma 4.10 and Lemma 4.15 for $S e(F, T, S)$. Namely, $f_{n^{2}}$ is the density with respect to Lebesgue measure of the law of $F_{n^{2}}:=\left(F \circ T^{m} \circ S^{n}\right)_{m, n=0, \ldots, n-1}$, and $h_{n}^{(2)}=-\log f_{n^{2}} \circ F_{n^{2}}$. The aim of this section is to prove the following theorem:

\section{Theorem 4.1:}

Let $T, S$ be commuting measure preserving transformations on the probability space $(\Omega, \mathcal{F}, \mu)$ with ergodic joint action. Let $F \in L^{2}(\mu)$ such that the process $\left(F \circ T^{m} \circ S^{n}\right)_{(m, n) \in \mathbb{Z}^{2}}$ is absolutely continuous, with law $\nu$. Let $\nu_{0}$ be the law of the process $\left(F \circ T^{m} \circ S^{n}\right)_{(m, n) \in \mathbb{N}^{2},(m, n) \neq(0,0)}$.

Assume that $S e(F, T, S)$ is finite (which is equivalent to $\left.H_{\mu F^{-1} \times \nu_{0}}(\nu)<\infty\right)$. Then $\frac{1}{n^{2}} h_{n}^{(2)}$ converges almost everywhere and in $L^{1}(\mu)$ to $\operatorname{Se}(F, T, S)$.

In case $\operatorname{Se}(F, T, S)=-\infty$ the previous convergence still holds almost everywhere.

Proof: We establish first the invariance of $\lim \inf \frac{1}{n^{2}} h_{n}^{(2)}$. Next, with the help of a reduction, we prove that this liminf is in fact almost everywhere a limit.

Recall that, for every $n, X_{n, n}^{n+1}=\left(x_{s, t}\right)_{s, t=0, \ldots, n}$ is, as in Section 4 , formula $\left(a_{0}\right)$.

(a): Let $h_{*}:=\liminf _{n} \frac{1}{n^{2}} h_{n}^{(2)}$. Then $h_{*}$ is invariant by each action.

Proof of (a): Let $K_{(0, n) \times(0, n)}:=X_{n, n}^{n+1}, K_{(1, n) \times(0, n-1)}:=\left\{x_{i, j}: 1 \leq i \leq n, 0 \leq j \leq n-1\right\}$ and $w_{n}=\frac{1}{n^{2}} h_{n}^{(2)}$. Then

$$
w_{n} \circ S-\frac{(n+1)^{2}}{n^{2}} w_{n+1}=y_{n}+z_{n}
$$

where $y_{n}:=\frac{1}{n^{2}} \log \frac{f\left(K_{(0, n) \times 0, n)}\right)}{\prod_{j=0}^{n} f\left(x_{0, j}\right) \times \prod_{i=1}^{n} f\left(x_{i, n}\right) \times f\left(K_{1, n) \times 0, n-1)}\right)}$, and $z_{n}:=\frac{1}{n^{2}} \log \left(\prod_{j=0}^{n} f\left(x_{0, j}\right) \times \prod_{i=1}^{n} f\left(x_{i, n}\right)\right)$. Now $z_{n}$ converges to 0 almost everywhere by the pointwise ergodic theorems. For the first one 
$y_{n}$, define, for $\epsilon>0, A_{n}(\epsilon)=A_{n}$ by

$$
A_{n}:=\left\{X: f\left(X_{n, n}^{n+1}\right) \leq e^{-n^{2} \epsilon} \prod_{j=0}^{n} f\left(x_{0, j}\right) \times \prod_{i=1}^{n} f\left(x_{i, n}\right) \times f\left(K_{(1, n) \times(0, n-1)}\right)\right\} .
$$

Then, a simple calculation yields $\mu\left(A_{n}\right) \leq e^{-n^{2} \epsilon}$ and thus for $\mu$ almost all $x$ there is $p$ such that $-y_{n}(x) \leq \epsilon$ for all $n \geq p$, and this implies $h_{*} \leq \epsilon+h_{*} \circ S$. Hence $h_{*} \leq h_{*} \circ S$. It follows that $h_{*}=h_{*} \circ S$.

In the same way, we have also $h_{*}=h_{*} \circ T$ and this proves (a).

We prove now that

(b) If $\lim _{n} \frac{1}{n^{2}} H_{n}^{(2)}$ is finite then the family $\left\{\frac{1}{n^{2}} h_{n}^{(2)}: n \geq 1\right\}$ is $\mu$ uniformly integrable.

Proof of (b): Recall that $\nu$ and $\mu$ are defined by their respective marginals $\nu_{n}, \mu_{n}$ as in (20) and (21) respectively, and $g_{i, j}^{n}$ is as in (32). Also $L$ is as in $\left(a_{2}\right)$ in subsection 4.1. We prove that the family $\left\{\log g_{i, j}^{n}:(i, j, n) \in L\right\}$ is $\mu$ uniformly integrable, and this will imply, by the equality (E) in Remark 4.12, that $\left(\frac{1}{n^{2}} h_{n}^{(2)}\right)$ is $\mu$ uniformly integrable. For $l=(i, j, n)$, denote $g_{i, j}^{n}$ by $\rho_{l}$, and let $l_{k}=\left(i_{k}, j_{k}, n_{k}\right)$, be an infinite sequence in $L$. We shall prove that $\left(\log \rho_{l_{k}}\right)$ contains a weakly convergent subsequence, and this proves (b). By Remark 4.1(2), $\left(l_{k}\right)$ contains a strictly increasing subsequence which we still denote $\left(l_{k}\right)$. Let $\mathcal{F}_{\infty}:=\vee_{k} \mathcal{F}_{l_{k}}$. By the formula (34) and Remark 4.13, $\rho_{l_{k}}$ converges $\nu$ almost everywhere to $\rho_{\infty}:=\frac{d \mu_{\infty}}{d \nu_{\infty}}$, where $\mu_{\infty}$ and $\nu_{\infty}$ are the restrictions of $\mu$ and $\nu$ to $\mathcal{F}_{\infty}$ respectively. Also we have

$$
0 \geq \int-\log \rho_{\infty} d \mu=-H_{\nu_{\infty}}\left(\mu_{\infty}\right) \geq-H_{\nu}(\mu)>-\infty
$$

That is $\log \rho_{\infty}$ is $\mu$ integrable.

But $\sup _{k} \int \rho_{l_{k}} \log \rho_{l_{k}} d \mu \leq \sup _{l} \int \rho_{l} \log \rho_{l} d \mu<\infty$. Hence, by Remark 4.14, $\left\{\left(\log \rho_{l_{k}}\right)^{+}: k \geq 1\right\}$ is uniformly integrable with respect to $\mu$. Set $Y_{k}=\log \rho_{l_{k}}$ and $Y=\log \rho_{\infty}$, so that $Y_{k}, Y \in L^{1}(\mu), Y_{k}$ converges $\mu$ almost everywhere to $Y$ and $\int Y_{k} d \mu$ converges to $\int Y d \mu$. It follows that $Y_{k}^{+}$converges $\mu$ almost everywhere to $Y^{+}$, and thus, because $\left(Y_{k}^{+}\right)$is $\mu$ uniformly integrable, the convergence holds in $L^{1}(\mu)$ too. In particular, $\int Y_{k}^{+} d \mu$ converges to $\int Y^{+} d \mu$. So $\int Y_{k}^{-} d \mu$ converges to $\int Y^{-} d \mu$, 
and thus, since $Y_{k}^{-}$converges $\mu$ almost everywhere to $Y^{-}$, it converges in $L^{1}(\mu)$. This proves that $\left(Y_{k}\right)$ converges in $L^{1}(\mu)$ and a fortiori it is $\mu$ uniformly integrable.

Now we proceed to prove that $\frac{1}{n^{2}} h_{n}^{(2)}$ converges $\mu$ almost everywhere. We begin by showing that

(c) We can reduce ourselves to the case where the density of the law of the first coordinate is greater than one on its support, and also where $\liminf _{n} \frac{1}{n^{2}} h_{n}^{(2)}<0$.

Proof of (c): Let $F: \mathbb{R}^{\mathbb{Z} \times \mathbb{Z}} \rightarrow \mathbb{R}$ be the projection to the $(0,0)$ coordinate, with absolutely continuous law with density $f_{0}=\frac{d \mu F^{-1}}{d \lambda}$, and $\alpha>0$. Let $\phi: \mathbb{R} \rightarrow \mathbb{R}$ be the map defined by

$$
\phi(x)=\frac{1}{\alpha} \int_{-\infty}^{x} f_{0}(t) d t
$$

Put $G=\phi \circ F$. Then the law of $G$ is absolutely continuous and has density $g_{0}$ given by $g_{0}=\alpha 1_{\left[0, \frac{1}{\alpha}\right]}$.

Let now $\Phi: \mathbb{R}^{\mathbb{Z} \times \mathbb{Z}} \rightarrow \mathbb{R}^{\mathbb{Z} \times \mathbb{Z}}$ be the map defined by: $(\Phi(x))_{i, j}=\phi\left(x_{i, j}\right), \forall i, j \in \mathbb{Z}$, so that $\Phi S^{m} T^{n}=$ $S^{m} T^{n} \Phi$, and let $m:=\mu \circ \Phi^{-1}$. Define $\theta$ by

$$
\theta(x)=\sup \{t \in \mathbb{R}: \phi(t)=x\}
$$

Then the finite dimensional marginals of $m$ are absolutely continuous with respect to Lebesgue measure and the following relationship holds between the densities $f$ for $\mu$ and $g$ for $m$ :

$$
g\left(\left(u_{i, j}\right)_{i, j=0, \ldots, n-1}\right)=f\left(\left(\theta\left(u_{i, j}\right)\right)_{i, j=0, \ldots, n-1}\right) \prod_{i, j=0, \ldots, n-1} \theta^{\prime}\left(u_{i, j}\right)
$$

with the property: for almost all $t, g_{0}(t)>0 \Rightarrow g_{0}(t) \geq \alpha$. It follows that

$$
-\frac{1}{n^{2}} \log \left(\left(u_{i, j}\right)_{i, j=0, \ldots, n-1}\right)=-\frac{1}{n^{2}} \log f\left(\left(\theta\left(u_{i, j}\right)\right)_{i, j=0, \ldots, n-1}\right)-\frac{1}{n^{2}} \sum_{i, j=0}^{n-1} \log \theta^{\prime}\left(u_{i, j}\right)
$$

But it is easy to see that $\log \theta^{\prime} \circ F \in L^{1}(m)$ if and only if $\int f_{0}(t) \log f_{0}(t) d t$ is finite. Also

$$
\int \log \theta^{\prime} \circ F d m=\log \alpha-\int f_{0}(t) \log f_{0}(t) d t
$$


So if $\int f_{0}(t) \log f_{0}(t) d t$ is finite, the sequence $\frac{1}{n^{2}} \sum_{i, j} \log \theta^{\prime}\left(u_{i, j}\right)=\frac{1}{n^{2}} \sum_{i, j} \log \theta^{\prime} \circ F \circ S^{i} T^{j}(u)$ converges $m$ almost everywhere to $\int \log \theta^{\prime} \circ F d m$. Therefore $-\frac{1}{n^{2}} \log g\left(\left(u_{i, j}\right)_{i, j=0, \ldots, n-1}\right)$ converges $m$ almost everywhere if and only if $-\frac{1}{n^{2}} \log f\left(\left(\theta\left(u_{i, j}\right)\right)_{i, j=0, \ldots, n-1}\right)$ does so. In this case the corresponding limits (or liminf), $h_{*}$ and $g_{*}$ verifiy

$$
g_{*}=h_{*}-\log \alpha+\int f_{0}(t) \log f_{0}(t) d t
$$

So if $h_{*}$ is $\geq 0$, then if we take $\alpha$ such that

$$
\log \alpha>h_{*}+\int f_{0}(t) \log f_{0}(t) d t
$$

we obtain $g_{*}<0$.

Therefore, we can suppose that for almost all $t$, if $f_{0}(t)>0$ then $f_{0}(t) \geq \alpha \geq 1$ and $h_{*}:=$ $\liminf \frac{1}{n^{2}} h_{n}<0$.

This finishes the proof of the announced reduction (c).

As in the Ornstein-Weiss case, we prove that

(d) $\liminf \frac{1}{n^{2}} h_{n}^{(2)}$ is almost surely a limit.

Proof of (d): Put $a:=h_{*}$. By (c), we can and do suppose that $a<0$. Let $\epsilon_{3}>0$, and $0<\epsilon<\epsilon_{3}$. Let $l_{0} \in \mathbb{N}, \epsilon_{1}, \epsilon_{2}>0$ and $\delta_{1}>0$, to be chosen later.

First, by the pointwise ergodic theorem, we find two sequences $\left(k_{l}\right)$ and $\left(m_{l}\right)$ of natural numbers converging to $\infty$ such that $\left(\frac{m_{l}}{k_{l}}\right)$ converges to $\infty$ as fast as we wish, a set $\Omega_{\epsilon}^{1} \subset \Omega$, with $\mu\left(\Omega_{\epsilon}^{1}\right)>1-\delta_{1}$, and an integer $N_{0}\left(\epsilon_{1}\right)$ such that

$$
\forall x \in \Omega_{\epsilon}^{1}, \forall N \geq N_{0}\left(\epsilon_{1}\right), \exists J_{N}(x) \subset I_{N-1, N-1}^{N}, \quad N^{2}\left(1-\delta_{1}-\epsilon_{1}\right) \leq \operatorname{card}_{N}(x),
$$

and

$$
\forall l, \forall(i, j) \in J_{N}(x), \exists n(i, j, l) \in\left[k_{l}, m_{l}\right], \quad e^{-n^{2}(i, j, l)(a+\epsilon)}<f\left((i, j)+X_{n(i, j, l)-1, n(i, j, l)-1}^{n(i, j, l)} .\right.
$$

Next, by repeated uses of a Vitali covering type Lemma, we get an upper estimate of the (Lebesgue) 
size of the set $\Omega_{\epsilon}^{1}$, which enables us to majorize the measure of the set

$$
\left\{x: f\left(X_{N-1, N-1}^{N}\right) \leq e^{-N^{2}\left(a+\epsilon_{3}\right)}\right\} \cap \Omega_{\epsilon}^{1},
$$

which will ensure the convergence of the series

$$
\sum_{N} \mu\left(\left\{x: f\left(X_{N-1, N-1}^{N}\right) \leq e^{-N^{2}\left(a+\epsilon_{3}\right)}\right\} \cap \Omega_{\epsilon}^{1}\right)
$$

in order to get $\lim \sup _{n} \frac{1}{n^{2}} h_{n}^{(2)} \leq h_{*}$. The details are as follows:

It is easy to see, from the definition of $a$, that, given $\epsilon_{0}>0$, there exist a set $\Omega_{\epsilon}$, with $\mu\left(\Omega_{\epsilon}\right) \geq 1-\epsilon_{0}$, and two strictly increasing sequences of natural numbers $k_{l}, m_{l}$, with $\frac{m_{l}}{k_{l}}$ converging to infinity as fast as one wishes, such that

$$
\forall l, \forall x \in \Omega_{\epsilon}, \exists n_{l}=n_{l}(x) \in\left[k_{l}, m_{l}\right], \quad e^{-n_{l}^{2}(a+\epsilon)}<f(x)=f\left(X_{n_{l}-1, n_{l}-1}^{n_{l}}\right) .
$$

Let $J_{N}(x):=\left\{(i, j): 0 \leq i, j<N ; T^{i} S^{j} x \in \Omega_{\epsilon}\right\}$. Then, by the pointwise ergodic theorem, for any $\delta>0$, there is a measurable set $A_{\delta}$ with $\mu\left(A_{\delta}\right)>1-\delta$, and a naural number $N_{0}\left(\epsilon_{1}, \epsilon, \delta\right)$, such that for all $N \geq N_{0}\left(\epsilon_{1}, \epsilon, \delta\right)$, and for all $x \in A_{\delta}$, it holds

$$
N^{2}\left(\mu\left(\Omega_{\epsilon}\right)-\epsilon_{1}\right)<\operatorname{card} J_{N}(x) \leq N^{2}\left(\mu\left(\Omega_{\epsilon}\right)+\epsilon_{1}\right) .
$$

Put $\delta_{1}=\epsilon_{0}+\delta$, and $\Omega_{\epsilon}^{1}:=\Omega_{\epsilon} \cap A_{\delta}$.

It follows $\mu\left(\Omega_{\epsilon}^{1}\right) \geq 1-\delta_{1}$, and for $x \in \Omega_{\epsilon}^{1}$ and $(i, j) \in J_{N}(x)$ there exists $n(i, j, l)=n(i, j, l)(x) \in$ $\left[k_{l}, m_{l}\right]$ such that

$$
e^{-n^{2}(i, j, l)(a+\epsilon)}<f\left((i, j)+X_{n(i, j, l)-1, n(i, j, l)-1}^{n(i, j, l)}\right)
$$

where we denoted $f\left(S^{i} T^{j} x\right)$ by $f\left((i, j)+X_{n(i, j, l)-1, n(i, j, l)-1}^{n(i, j l)}\right)$. 
Note that $R_{i, j, l}(x):=(i, j)+X_{n(i, j, l)-1, n(i, j, l)-1}^{n(i, j, l)}$ is the square with first vertex $(i, j)$ and with side having length $n(i, j, l)$. Then, for any $l,\left\{R_{i, j, l}(x):(i, j) \in J_{N}(x)\right\}$ is a finite cover of $J_{N}(x)$ by squares. So, due to the freedom, mentioned above, in the choice of $k_{l}$ and $m_{l}$, by repeated applications of the Vitali covering Lemma,[Mattila], for any $\epsilon_{2}$ there is $N_{1}\left(\epsilon_{2}, \epsilon\right)$ and $l_{1}, \ldots, l_{k} \geq l_{0}$, such that for any $N \geq N_{1}\left(\epsilon_{2}\right)$, there exist subsets $J_{N, l_{1}}(x), \ldots, J_{N, l_{k}}(x)$ of $J_{N}(x)$, such that the squares $\left\{R_{i, j, l_{s}}(x):(i, j) \in J_{N, l_{s}}(x), s=1 \ldots k\right\}$ are disjoint, and there is a subset $J_{N}^{0}(x) \subset J_{N}(x)$ which is covered by $\left\{R_{i, j, l_{s}}(x):(i, j) \in J_{N, l_{s}}(x), s=1 \ldots k\right\}$ and with $\left(1-\epsilon_{2}\right) \operatorname{card} J_{N}(x) \leq \operatorname{card} J_{N}^{0}(x)$. It follows that, for $\left.N \geq N_{0}\left(\epsilon_{1}, \epsilon, \delta\right)\right) \vee N_{1}\left(\epsilon_{2}, \epsilon\right)$ and $x \in \Omega_{\epsilon}^{1}$, we have

$$
N^{2} u \leq \operatorname{card} J_{N}^{0}(x)
$$

where $u:=\left(1-\epsilon_{2}\right)\left(1-\delta_{1}-\epsilon_{1}\right)$. Then

$$
N^{2} u \leq \sum_{s=1}^{k} \sum_{(i, j) \in J_{N, l_{s}}} n^{2}\left(i, j, l_{s}\right)(x)
$$

Thus, since $a<0$, if $\epsilon>0$ is chosen such that $a+\epsilon<0$,

$$
N^{2} u(a+\epsilon) \geq \sum_{s=1}^{k} \sum_{(i, j) \in J_{N, l_{s}}} n^{2}\left(i, j, l_{s}\right)(x)(a+\epsilon)
$$

Let $J_{N}^{1}(x)$ be the intersection with $I_{N-1, N-1}^{N}$ of the union of the squares $\left\{R_{i, j, l_{s}}(x):(i, j) \in\right.$ $\left.J_{N, l_{s}}(x), s=1 \ldots k\right\}$. Then if $(i, j) \notin J_{N}^{1}(x)$ we have that $1 \leq \frac{1}{\alpha} f\left(x_{i, j}\right)$. In particular

$$
\alpha \geq 1 \Rightarrow 1 \leq f\left(x_{i, j}\right), \forall(i, j) \notin J_{N}^{1}(x) .
$$

Since $\alpha \geq 1$, it follows, by (59), that

$$
1 \leq e^{\sum_{s=1}^{k} \sum_{(i, j) \in J_{N, l_{s}}(x)} n^{2}\left(i, j, l_{s}\right)(x)(a+\epsilon)}\left(\prod_{s=1, \ldots, k,(i, j) \in J_{N, l_{s}}(x)} f\left(R_{i, j, l_{s}}(x)\right)\right) \times \prod_{(i, j) \notin J_{N}^{1}(x)} f\left(x_{i, j}\right) .
$$


So by (61), we get for $x \in \Omega_{\epsilon}^{1}$,

$$
1 \leq e^{N^{2} u(a+\epsilon)}\left(\prod_{s=1, \ldots, k,(i, j) \in J_{N, l_{s}}(x)} f\left(R_{i, j, l_{s}}(x)\right)\right) \times \prod_{(i, j) \notin J_{N}^{1}(x)} f\left(x_{i, j}\right) .
$$

But the number of all configurations of such disjoint squares is majorised by $C_{N^{2}}^{\left[N^{2} \beta\right]}$, which is majorised by $c e^{N^{2} h(\beta, 1-\beta)}$, where $\beta=\frac{1}{q^{2}}, q$ being the smallest $k_{l}$ 's, and $c$ is a constant. Then by (62), the Lebesgue measure $\lambda\left(\Omega_{\epsilon}^{1}\right)$ of $\Omega_{\epsilon}^{1}$ is, for $N$ big enough, majorized by $c e^{N^{2} h(\beta, 1-\beta)} \times e^{N^{2} u(a+\epsilon)}$. But easily,

$$
\mu\left(\left\{f\left(X_{N-1, N-1}^{N}\right) \leq e^{-N^{2}\left(a+\epsilon_{3}\right)}\right\} \cap \Omega_{\epsilon}^{1}\right) \leq e^{-N^{2}\left(a+\epsilon_{3}\right)} \lambda\left(\Omega_{\epsilon}^{1}\right) .
$$

So for $N$ big enough, we then obtain $\mu\left(\left\{f\left(X_{N-1, N-1}^{N}\right) \leq e^{-N^{2}\left(a+\epsilon_{3}\right)}\right\} \cap \Omega_{\epsilon}^{1}\right) \leq c e^{-N^{2} \omega}$, where $\omega=a+\epsilon_{3}-h(\beta, 1-\beta)-u(a+\epsilon)$. But there is a constant $\gamma>0$, such that for $N$ big enough, the exponent $\omega$ is $>\gamma$. In fact, if we put $v=\epsilon_{1}+\delta_{1}$, then $u=1-v-\epsilon_{2}(1-v)$, and thus $\omega=\epsilon_{3}-\epsilon-h(\beta, 1-\beta)+(a+\epsilon)\left(v+\epsilon_{2}(1-v)\right)$, so that, for $0<\gamma<\frac{\epsilon_{3}-\epsilon}{2}$, we can choose $\epsilon_{1}, \epsilon_{2}$, $\delta_{1}, l_{0}$ and $N_{2} \geq N_{0} \vee N_{1}$, such that $\forall N \geq N_{2}$, we have the inequality

$$
\epsilon_{3}-\epsilon>2 \gamma>\gamma>h(\beta, 1-\beta)-(a+\epsilon)\left(v+\epsilon_{2}(1-v)\right)
$$

Then the series

$$
\sum_{N} \mu\left(\left\{f\left(X_{N-1, N-1}^{N}\right) \leq e^{-N^{2}\left(a+\epsilon_{3}\right)}\right\} \cap \Omega_{\epsilon}^{1}\right)
$$

is convergent. Letting $\delta_{1} \rightarrow 0$, we get $\limsup _{N} w_{N}(x) \leq a+\epsilon_{3}$, and finishes the proof in the case $-\infty<a:=\liminf _{N} w_{N}<0$.

If $\lim \inf w_{N}=-\infty$, taking $a$ any negative number, the same proof gives $\lim \sup _{N} w_{N} \leq a$. This finishes the proof of theorem 4.

\section{References}

[1] Barron, A.R. : The strong ergodic theorem for densities: Generalized Shannon-McMillanBreiman theorem. Ann. of Prob., (1985), Vol. 13, No. 4, 1292-1303. 
[2] Doob, J. L. : Stochastic processes. Wiley, New York, 1953.

[3] Dye, H. A. : On groups of measure preserving transformations, I. Amer. J. Math., 81, 1959, $119-159$.

[4] Helson, H. : Harmonic Analysis. Addison-Wesley (1983).

[5] Hoffman, K.: Banach Spaces of Analytic Functions. Prentice-Hall (1962).

[6 ] Hoffman, C., Rudolph, D. J. : Uniform endomorphisms which are isomorphic to Bernoulli shift. Ann. of Math. (2), (2002), 156, No. 1, 79-101.

[7] Kolmogorov, A. N. : Stationary sequences in Hilbert space. Bull. Math. Univ. Moscow 2, no 6 (1941).

[8] Mattila P. : Geometry of Sets and Measures in Euclidean Spaces, Fractals and rectifiability. Cambridge Studies in Advanced Mathematics, 44. Cambridge university Press, Cambridge 1995.

[9] Ornstein, D. S. : Ergodic Theory, Randomness, and Dynamical Systems. Yale University Press (1974).

[10] Ornstein, D. S., Weiss B. : The Shannon-Mc Millan-Briman Theorem For A Class Of Amenable Groups. Isr. J. Math. Vol. 44, Mo. 3, 1983, pp. 53-60. 
[11] Parry, W. : Entropy and Generators in Ergodic Theory. W.A. Benjamin (1969).

[12] Parry, W. : Topics in Ergodic Theory. Cambridge University Press (1981).

[13 ] Parry,W. : Automorphisms of the Bernoulli endomorphism and a class of skew-products. Erg. Th. and Dyn. Syst. 16 (1996), 519-529.

[14] Pinsker,M.S. : Information and Information Stability of Random Variables and Processes. Holden-Day (1964).

[15] Rosenblatt, M. : Stationary processes as shifts of functions of independent random variables. J. Math. Mech. 8 (1959) 665-681.

[16] Shiriayev, A.N. : Probability. Second edition, Springer-Verlag (1989).

[17] Shannon, C.E. : The mathematical theory of communication, Bell System Tech. J. 27 (1948), 379-423; 27 (1948), 623-656.

[18] Simon, B. : The Sharp Form of the Strong Szegö Theorem, To appear in Proc. Conf. on Geometry and Spectral Theory.

[19] Smorodinsky, M. : Ergodic Theory, Entropy. Lecture Notes, 214, (1971), Springer-Verlag.

[20] Wiener, N. : Extrapolation, interpolation and smoothing of stationary time series. New York, 
Wiley (1945).

[21] Wiener, N. : Non-linear problems in random theory. MIT Press, Cambridge, Mass., and Wiley, New York (1958). 